\title{
Research Square \\ Cybersecurity Threats Detection In loT Using Krill Based Deep Neural Network Stacked Auto Encoders
}

Pragati Rana ( $\Delta$ pragthiana20@gmail.com )

Army institute of technology

\section{Sanjeev Chauhan}

Microsoft

B P Patil

army Institute of technology

\section{Research Article}

Keywords: Data pre-processing, Feature extraction, features selection, optimization, cybersecurity threats detection.

Posted Date: April 14th, 2021

DOI: https://doi.org/10.21203/rs.3.rs-349982/v1

License: @ (i) This work is licensed under a Creative Commons Attribution 4.0 International License. Read Full License 


\author{
Cybersecurity Threats Detection In Iot Using Krill Based Deep Neural Network \\ Stacked Auto Encoders \\ ${ }^{1}$ Pragati Rana, ${ }^{2}$ Sanjeev Chauhan, ${ }^{3}$ B P Patil \\ ${ }^{I}$ Assistant Professor, Department of Electronics and Telecommunication, Army Institute of Technology, Pune \\ ${ }^{2}$ SDE3, Microsoft, Noida, India \\ ${ }^{3}$ Principal, Army Institute of Technology, Pune. \\ ${ }^{1}$ Email id: pragthiana20@gmail.com
}

\begin{abstract}
The Internet of things (IoT) has concerned much significance for some manufacturing sectors including clinical fields, co-ordinations following, savvy urban communities, and automobiles. Anyway as a worldview, it is sensitive to different sorts of cyber-attacks. Customary very good quality security resolutions for guarantee an IoT structure are not reasonable. This deduces clever organization-based security plans as AI arrangements ought to be made. In this work, we propose Cyber Security Threats recognition in IoT utilizing Krill Based Deep Neural Network Stacked Auto Encoders (KDNN-SAE). In our proposed approach, first, the information pre-processing measure was acted in the underlying development before isolating the dataset into two segments: preparing and test. At that point, flow-based features are extracted from the pre-processed information. By then, the properties to be utilized by the algorithms are chosen in the attribute determination utilizing the Genetic Algorithm (GA). At last, our methodology completes with the execution of the machine learning algorithm KDNN-SAE. The exploratory results show that the introduced method beats the existing techniques to different execution measures.
\end{abstract}

Keywords: - Data pre-processing, Feature extraction, features selection, optimization, cybersecurity threats detection.

\title{
1. INTRODUCTION
}

In the modern years, deep learning has developed a significant strategy in numerous informatics fields, for example, visual identification, natural language processing, and bioinformatics [1-3]. As developing innovation achievements, IoT has empowered the assortment, treating, and correspondence of information for smart purposes [4]. Cyber-attack is a basic issue at the point of the IoT [5]. The IoT characterizes a hopeful future, where the items will have the option to use the Internet and make knowledgeable coordinated efforts with one another everywhere and whenever $[6,7]$.

Most of the time, new processing tool is sold with pirated software that contains attack. Attack packaged with pirated software is one of the essential customs by which PCs are ruined [8]. Additionally, the threat may infect processing tools from pilfered programming downloaded through the web or bought from sellers. In these serious threats, obscure malware that has not been controlled by security sellers is frequently utilized for evading the malware recognition framework $[9,10]$.

Furthermore, deep learning has been applied to network security recognition [11]. Deep learning is an information portrayal and learning technique dependent on machine learning. Tensor Flow is a profound learning open-source documentation made by Google Inc [12]. It is an open-source man-made intellectual documentation, utilizing data flow charts to fabricate designs. It permits designers to make the enormous scope of neural frameworks with various layers. Tensor Flow is primarily utilized for: Classification, Perception, Perceptive, Discovering, Prediction, and Formation [13, 14]. On the opposite side, code initiation attribution assumes an important job in software forensics activities, security examination, and software plagiarism detection [15] particularly for focusing on malware 
creators. The malware writers compose malicious software that can co-operate the compilation method in the PC system $[16,17]$.

Even though there are numerous public C++ laboratory datasets, the Google Code Jam 1 dataset is most probably the greatest of all. Tests from this dataset are gathered to research software piracy [18]. As one of the significant models in profound learning, a convolutional neural framework (CNN) [19] has been prominently utilized for recognition and demonstrated promising execution in relevant classification. Further, the deep convolution neural system is utilized to catch the malicious patterns of malware through binary visualization [20]. The primary commitments of this paper are as per the following,

$>$ Pre-processing is the initial step separating the dataset keen on training and testing sets.

$>$ Effective flow-based features are extracted from the pre-processed information.

$>$ Optimum features are selected utilizing the Genetic Algorithm (GA).

$>$ Cyber Security Threats recognition in IoT is achieved utilizing Krill Based Deep Neural Network Stacked Auto Encoders (KDNN-SAE).

The configuration of the composition is devised as follows: Section 2 discusses the related works to the presented method. In section 3, a short explanation about the presented structure is specified, section 4 decides the analyzing results, and section 5 concludes the paper.

\section{RELATED WORK}

Jonghoon Lee et al. [21] proposed a Man-made intelligence strategy for cyber-threats recognition, given artificial neural organizations. The proposed procedure changes a huge number of gathered safety measures to singular events summary and utilizes a profound learning-based recognition technique. They built up an AI-SIEM framework dependent on an arrangement of event summary for information pre-processing and distinctive neural organization strategies, comprising FCNN, CNN, and LSTM in this work. The scheme centers on isolate among genuine positive and bogus positive cautions, subsequently serving security predictors to quickly retort to cyber threats. Yin Chuan-long et al. [22] presented a profound learning strategy for interference acknowledgment using intermittent neural organizations (RNN-IDS). Additionally, they thought about the introduction of the technique in double characterization and assorted learning rate impact on the introduction of the presented strategy. They differentiated it and those of current AI methodologies presented through before specialists on the standard dataset. Zhihua $\mathrm{Cu}$ et al. [23] presented a new technique that utilized deep learning to enhance the recognition of defect variations. In an earlier examination, deep learning exhibited great execution in image recognition. To actualize their proposed recognition strategy, they transformed the malicious code into grayscale images. They were perceived and masterminded to use a CNN might eliminate the highlights of the malware ordinarily. Additionally, they utilized a bat design to manage the data indiscretion between numerous malware relatives. Michal Choras et al. [24] concentrated on oppose rising application layer threats as those are recorded as pinnacle dangers and the guideline issues for the framework and network safety. The huge duty of the examination is the recommendation of an AI technique to manage the model's conventional direct of capacity and to distinguish digital threats. The model involves plans that are procured using diagram-based division methods and powerful programming. The model relies upon information got from HTTP demands requests by the client to a web worker. Fanzhi Meng et al. [25] proposed a new trait grouping insider threat recognition technique dependent on long transient memory repetitive neural organizations (LSTM-RNNs) to recognize malignant insiders. To achieve a high acknowledgment rate, occasion authority, highlight extractor, a few component classifiers, and difference number crunchers are reliably 
organized into a start to finish acknowledgment structure. By the CERT insider danger dataset v6.2 and danger disclosure survey as presentation metric, test outcomes favor that the presented danger acknowledgment procedure altogether defeats the current analysis dependent hazard identification techniques.

\section{PROPOSED METHODOLOGY}

Cyber Security Threats recognition in IoT utilizing Krill Based Deep Neural Network Stacked Auto Encoders (KDNN-SAE) is introduced in this work. In the presented technique, the raw dataset is apportioned into preparing and testing data as a data pre-processing step. In this way, flow-based features are separated from the training and testing data. At that point, the removed features are chosen using the Genetic Algorithm (GA). At long last, our methodology ends with the usage of the machine learning algorithm KDNN-SAE. Here the krill herd optimization is utilized to develop the loads utilized in the deep neural network stacked auto-encoders. The flow representation of the presented methodology is shown in figure 1.

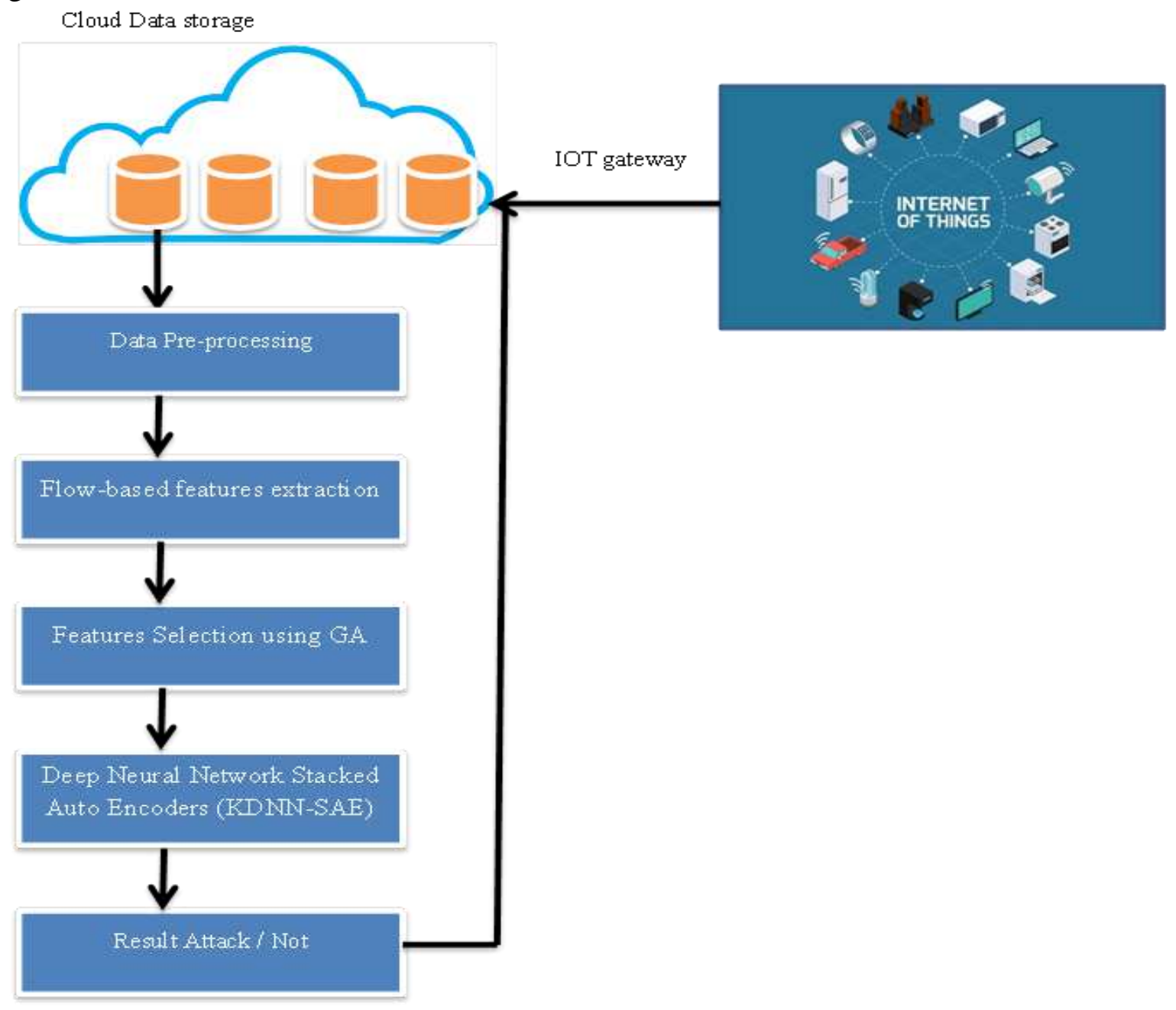

Figure 1: The illustration of the presented approach

\subsection{DATA PRE-PROCESSING}

Pre-processing method is accomplished in the initial stage for separating the input dataset into two sections are training and testing information. In this way, flow-based features are removed for the Cyber Security Threats detection in IoT.

\subsection{FLOW BASED FEATURES EXTRACTION}


Flow is characterized by a progression of information packages with comparable characteristics. By Tor traffic, each flow is TCP, meanwhile, it doesn't maintain UDP. Close by the streaming age, we process the highlights identified with each stream [28]. In this way we have a rundown and clarification of the features assessed qualities: fiat, biat, flowiat, dynamic, inactive, fb psec, fp psec, duration around six gatherings of features. The underlying three gatherings are explicit: - fiat, - biat, and - flowiat, and are pointed exclusively on the onward, in reverse, and bi-directional flows. The fourth and fifth gatherings of features are resolved to the inactive to-dynamic or dynamic to-sit states and are termed - inactive and - dynamic. Lastly, the keep going gathering centers on the size and quantity of packages every second is termed - psec feature.

\subsection{Features selection using a genetic algorithm (GA)}

All the extricated features don't give exact recognition results. Consequently, it is fundamental to arrange the most separating features before the recognition method for appropriate outcomes. In this paper, feature selection is completed by utilizing a GA [29]. The pseudo-code of the GA is given in algorithm 1.

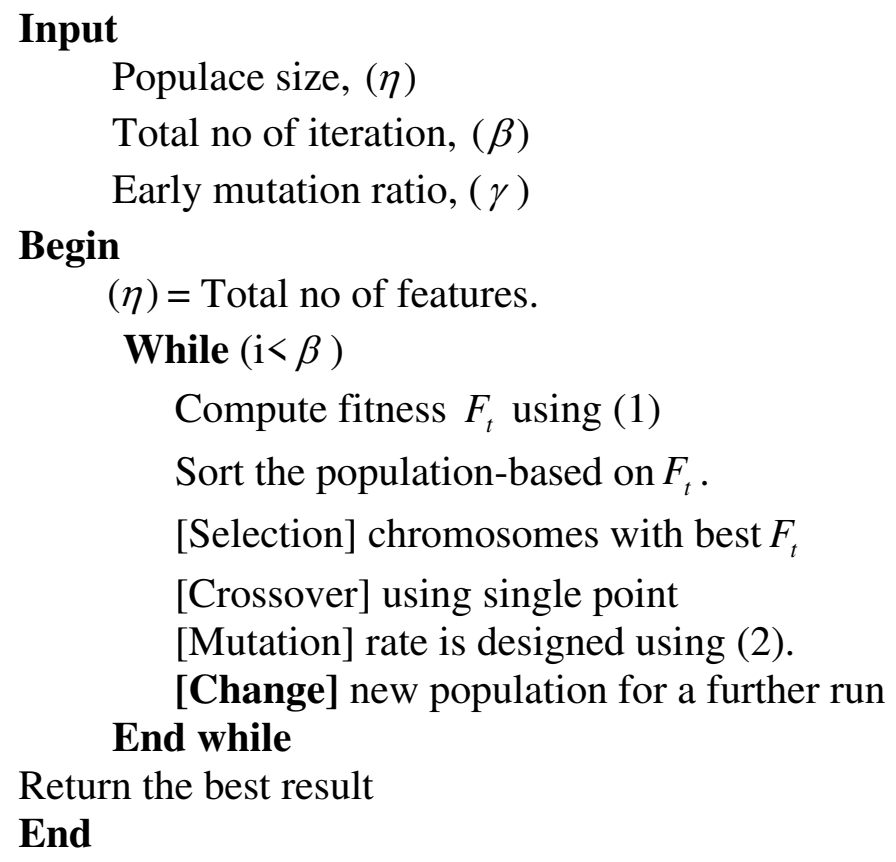

Algorithm 1: Pseudo-code of Genetic Algorithm

The steps of the GA are described in the subsequent steps,

Step 1: To begin with, the number of $(\eta)$ and $(\beta)$ is set. The $(\eta)$ size means the number of features chosen.

Step 2: The fitness $F_{t}$ is resolved using equation (1).

$$
F_{t}=1-\left(\frac{1}{n_{d}} \sum_{i=1}^{n_{d}}\left(z_{i}-\hat{z}_{i}\right)^{2}\right)
$$

Step 3: Arrange the fitness and their relating chromosomes.

Step 4: Choose the chromosomes with the best $F_{t}$ values. The first half of the population is chosen here.

Step 5: A one-point cross is executed on the chosen parent chromosomes.

Step 6: The mutation rate is determined by (2), 


$$
M_{R}=1+B * \frac{\left(f_{\max }-f_{\min }\right)^{n c}-\left(f_{\text {avg }}\right)^{n c}}{S_{i} \times\left(f_{\max }-f_{\min }\right)^{n c}-\left(f_{\text {avg }}\right)^{n c}}
$$

In which $n c$ is the population of the chosen parent chromosomes, $f_{\min }$ is the worst fitness value, $f_{\max }$ signifies the finest fitness esteem, $f_{\text {avg }}$ signifies the normal fitness esteem, $B$ is taken as 2 , and $S_{i}$ is the control parameter given by equation (3).

$$
S_{i}=\left(\frac{f_{\max }-f_{\min }}{f_{\text {avg }}}\right)^{n c}
$$

This difference in the mutation rate in GA is the use of best results is improved, subsequently, accelerating the assembly and avoiding the population from being caught at the local minima majority of the time.

\subsection{KRILL BASED DEEP NEURAL NETWORK STACKED AUTO ENCODERS (KDNN-SAE)}

\subsubsection{Auto-Encoder}

The encoder consists of an input and concealed layer, here the unique informational index $(A)$ is biased and plotted to acquire a deterministic plot $B^{\prime}$ :

$$
B^{\prime}=\tilde{\sigma}\left(\tilde{W}_{m}{ }^{T} A^{\prime}+\hat{b}\right)
$$

Here, $\tilde{\sigma}$ represents a sigmoid function, $A^{\prime}$ represents an input matrix, $\tilde{W}_{m}$ represents a weight matrix, and $\hat{b}$ represents an $m$-dimensional balance vector. The purpose of the encoder is to pack more significant level information into lower-level information.

$$
C^{\prime}=\tilde{W}_{m} B^{\prime}+\hat{b}^{\prime}
$$

Here, $C^{\prime}$ represents an activation function, $\tilde{W}_{m}$ represents the restoration decoder's weights matrix, and $\hat{b}$ represents the remaking m-dimensional balance vector. The purpose of the decoder is to recreate lesser-level information. At last to make the information esteems and the yield esteems as same as plausible the 2 sets $\left(\tilde{W}_{m}, \hat{b}\right)$ and $\left(\tilde{W}_{m}{ }^{\prime}, \hat{b}^{\prime}\right)$ is repeated targeting to reducing the reconstruction error $\bar{L}\left(A^{\prime}, C\right)$. It is characterized as,

$$
\bar{L}\left(A^{\prime}, C^{\prime}\right)=\frac{1}{2}\left(A^{\prime}-C^{\prime}\right)^{2}
$$

When an autoencoder (AE) consists of an enormous quantity of hidden layer neurons, even though the computation accurateness is enhanced, the over-fitting issue also happens, and the system may just get familiar with the rehashed illustration of the original data. To conquer these issues presented the subsequent system with AE.

\subsubsection{Deep Learning with Stacked De-noising Auto Encoder (SDAE)}

Deep learning with stacked de-noising AE debases the input information to avoid the issues in the given $\mathrm{AE}$. The defilement is to degenerate the input information $\left(X^{\prime}\right)$ to explicit extents. From this point onward, the ruined information is packed and reproduced to create the input information close to the yield information.

Nonetheless, in various training assignments, small organization (for example, DAE) capacities are restricted and regularly don't present very much contrasted with DNNs. An SDAE is loaded via various DAEs. Initially, input information is utilized to create an advanced illustration. Then, the concealed layer is considered as the contribution of the following DAE to extricate advanced illustrations. As seemed in figure 2, the input of the 
following DAE is $\mathrm{Y}$. It is compacted, and reproduced to acquire $\mathrm{Y}^{2}$. Similarly, SDAE is constructed by loading numerous DAEs as appeared in Figure 2.

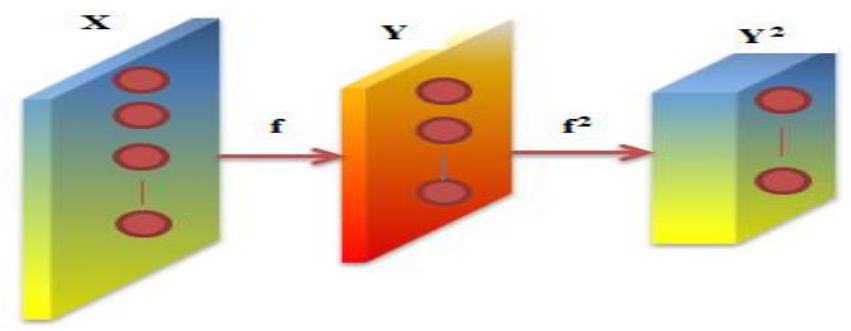

Figure 2: Structure of SDAE

The presented network is used to choose the best patches and it is viably made with the enthalpy-based normalization and diminishes the time complication in deep learning. The proposed DNN classifier contains layers, for example, convolution, enthalpy-based normalization, pooling, and fully connected layer. The flow representation of a presented KDNN-SAE is given in figure 3.

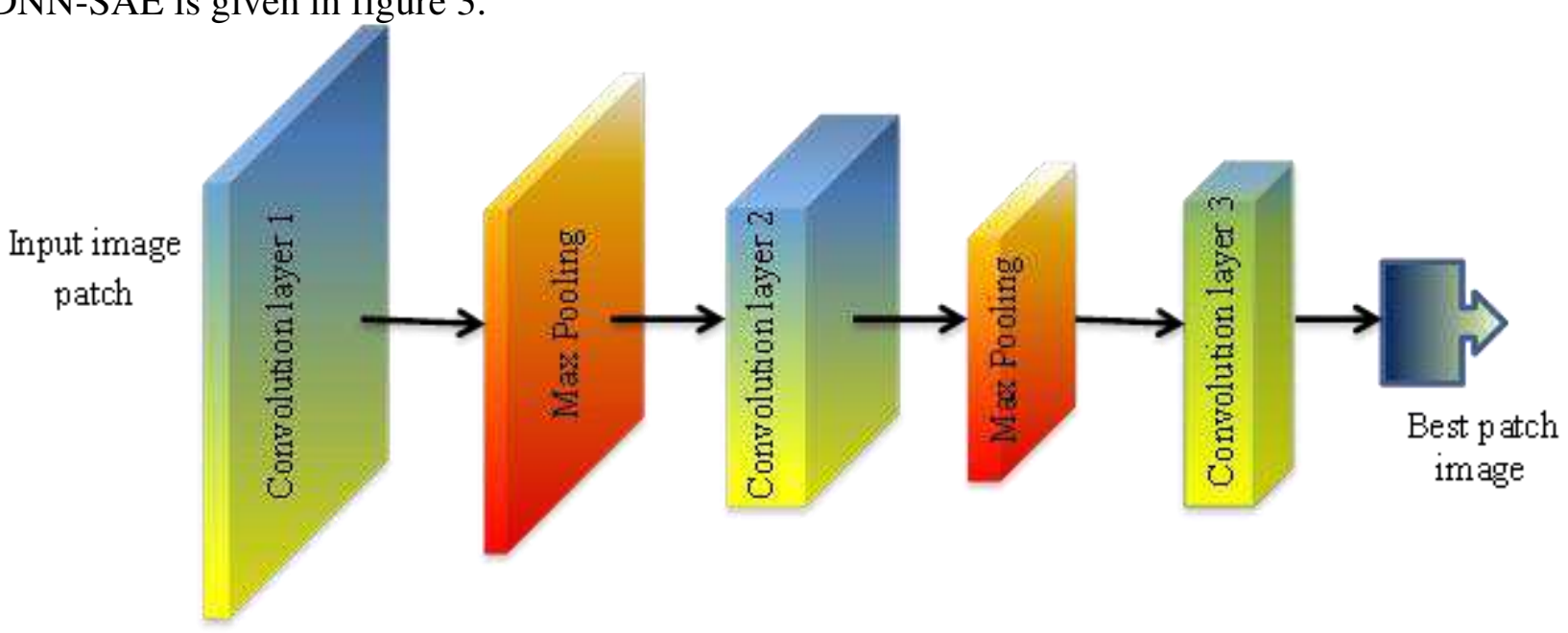

Figure 3: Presented KDNN-SAE system

The DNN classifier is completing decision depends upon the weights and inclinations of the earlier layers in the design architecture. Here, the weight function represented in condition (5) is improved using the krill herd optimization algorithm.

\subsubsection{KRILL HERD OPTIMIZATION (KHO)}

In KHO algorithm utilized a useful fitness function to enhance its consistency and quality managing enhancement problems. The pseudo-code of $\mathrm{KHO}$ is mentioned in algorithm 2 .

\section{Step 1}

At first, the selected features are initialized.

\section{Step 2}

The fitness esteem is evaluated for each krill as specified through the determined entropy measure. The greatest entropy esteem is picked as fitness.

\section{Step 3}

Subsequent, the vital iteration of optimization starts via essentially classifying the krill from maximum to the highest discernibly terrible individual.

\section{Step 4}


Subsequently, development updates are determined for every krill using the subsequent conditions.

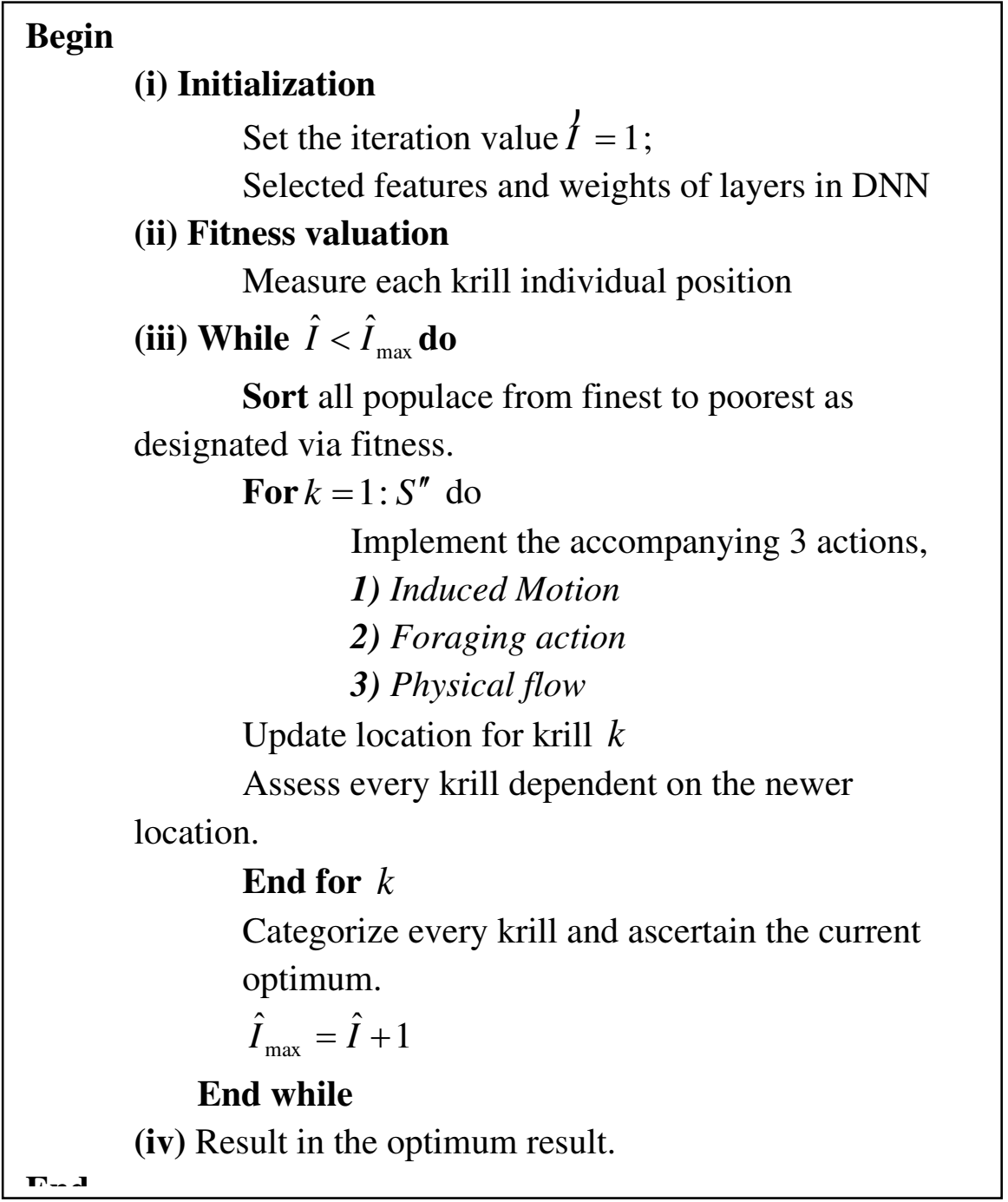

Algorithm 2: Pseudocode for KHO

\section{a) Foraging motion}

This is upgraded via the subsequent conditions,

$$
\begin{gathered}
\tilde{M}_{N}(\hat{t}+1)=F^{\prime}{ }_{S} \bar{\beta}_{N}+\omega_{i}^{\prime} \tilde{M}_{N}(\hat{t}) \\
\bar{\beta}_{F}=\bar{\beta}_{F}^{\text {food }}+\bar{\beta}_{F}^{\text {best }}
\end{gathered}
$$

Here, $\tilde{M}_{s}$ represents the foraging speed, $\omega_{i}^{\prime}$ represents the inertia weight, $\bar{\beta}_{N}^{\text {food }}$ represents the food attractive, and $\bar{\beta}_{N}^{\text {best }}$ represents the greatest solution for the $N^{\text {th }}$ krill individual.

\section{b) Induced movement}

This is upgraded for every krill is computed as,

$$
\begin{gathered}
\tilde{I}_{N}(\hat{t}+1)=I_{S}^{\prime} I_{\max }^{\prime} \bar{\alpha}_{N}+\omega_{i}^{\prime}+I^{\prime}{ }_{N}(\hat{t}) \\
\bar{\alpha}_{N}=\bar{\alpha}_{N}^{\text {local }}+\bar{\alpha}_{N}^{t \operatorname{tag} e t}
\end{gathered}
$$


Where, $I_{S}^{\prime}$ signifies the maximum induced speed, $\omega_{i}^{\prime}$ signifies the inertia weight, $\bar{\alpha}_{a}^{\text {total }}$ signifies the local impact of the $N^{\text {th }}$ krill on neighborhoods, $\bar{\alpha}_{N}^{t \text { arg et }}$ signifies the optimum arranging of $N^{\text {th }}$ krill.

\section{c) Physical diffusion}

This apprises is corresponding the physical propagation via haphazard action is signified as,

$$
D_{N}^{\prime}(\hat{t}+1)=D^{\prime}{ }_{S}\left(\frac{1-i^{\prime}}{i_{\text {max }}^{\prime}}\right) \delta^{\prime}
$$

Here, $D^{\prime}{ }_{S}$ signifies the very excessive diffusion rapidity in $[-1,1]$.

\section{Step 5}

Given the recently referenced actions, using recognizing boundaries of action amidst time, the location of $a^{\text {th }}$ krill in time $\hat{t}^{\prime}+\Delta \hat{t}^{\prime}$ to be expressed through the following condition and this is used to figure krill location.

$$
K_{N}^{\prime}\left(\hat{t}^{\prime}+\Delta \hat{t}^{\prime}\right)=K_{N}^{\prime}\left(\hat{t}^{\prime}\right)+\Delta \hat{t}^{\prime} \frac{d K_{N}^{\prime}}{d \hat{t}^{\prime}}
$$

Where $\Delta \hat{t}^{\prime}$ are fundamental coefficients which would be modified greatest. By using the previously represented condition, the location of the krill is referenced for assessing the krill target work at the end of the optimization, the utmost excellent krill is restored.

\section{Step 6}

Lastly, the ending state is exploited for the fulfilment of a pre-assigned quantity of function evaluations. Though the ending basis is not met another time, arrange the krill populace from most excellent to generally undesirable and then determine the motion apprises for every krill and audit the krill location. This restores the most excellent krill when the condition meets. The schematic representation of KHO is presented in figure 4.

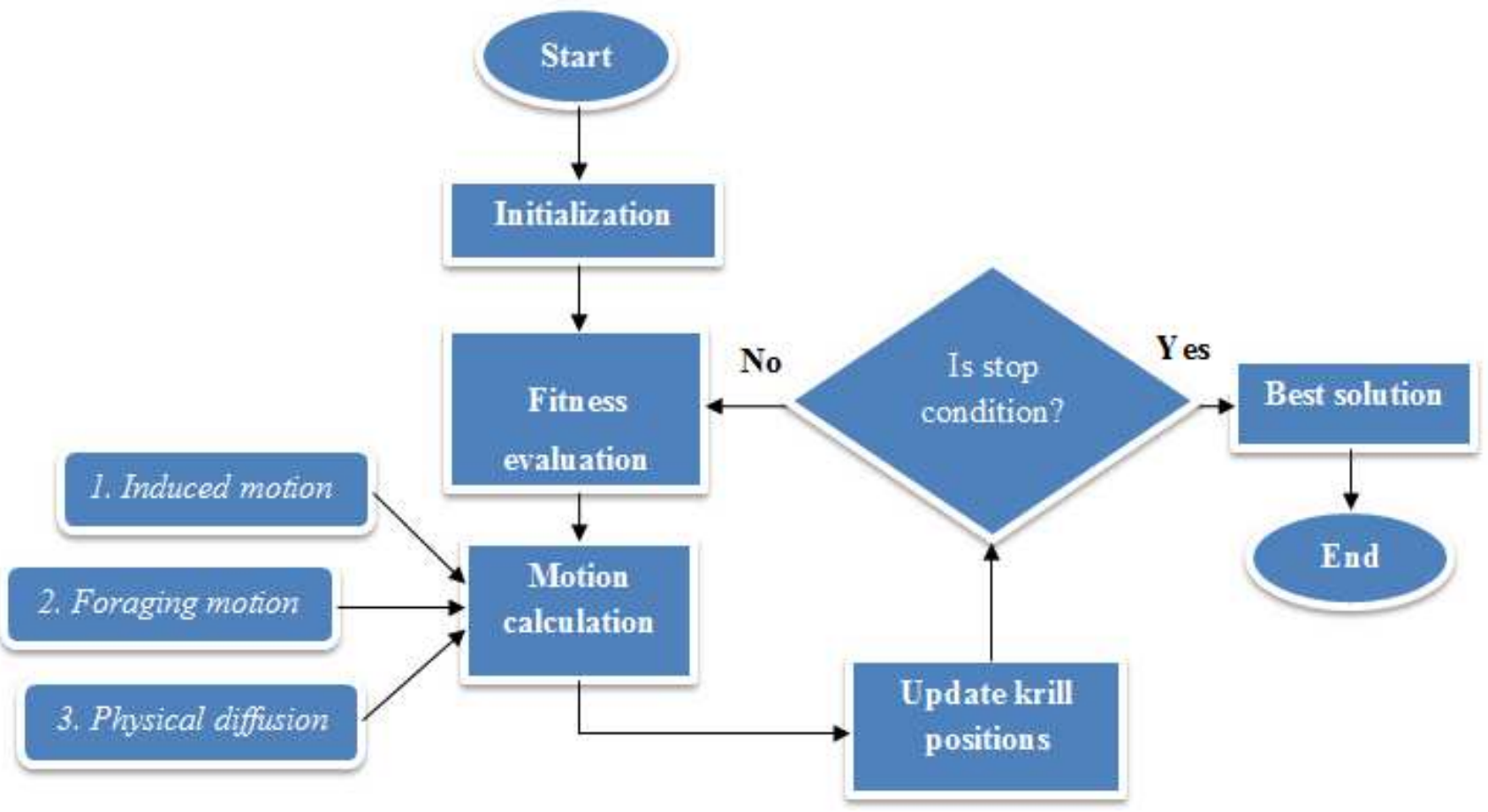

Figure 4: Flow representation of KHO 
The krill herd optimization results in the optimized weights to the proposed system. Thus, the model is updated in conditions (13) and (14) separately for all layers.

$$
\begin{gathered}
\Delta W_{l}=-\frac{x \lambda}{r} W_{n}-\frac{x}{N_{t}} \frac{\partial C}{\partial W_{n}}+m \Delta W_{n}(\hat{t}) \\
\Delta B_{n}=-\frac{x}{n} \frac{\partial C}{\partial B_{n}}+m \Delta B_{n}(\hat{t})
\end{gathered}
$$

Where, $W_{n}$ signifies the weight, $B_{n}$ signifies the bias, $n$ signifies the layer number, $\lambda$ signifies the regularization parameter, $x$ signifies the learning rate, $N_{t}$ signifies the sum of preparation samples, $m$ signifies the momentum, $t$ signifies the modernizing time, and $C$ signifies the cost-utility. The DNN classifier comprises of different sorts of layers are as per the following,

(a) Convolutional layer: It includes many scholarly weight matrices called filters that drop above the input information and finishes the convolution of the input information with the kernel by using equation (15). The outcome is also considered as the feature map.

$$
C_{k}=\sum_{m=0}^{M-1} y_{n} \hat{h}_{k-n}
$$

Where $y_{n}$ indicates the reproduced low feature value, $\hat{h}$ represents the filter, and $M$ represents the number of components in $y$ and the output vector is $C_{k}$.

(b) Pooling layer: It reduces the dimension of resultant neurons from the previous layer to decrease the estimation power and maintain a strategic distance from the overfitting. This selects the maximum exceptional motivator in all components and therefore, diminishing the measure of output neurons.

(c) Fully connected layer: It is a complete connection with all initiation in the previous layer. That connects every neuron from the most extreme pooled layer to the majority of output neurons. The activation function is according to the subsequent,

Softmax: This evaluates the likelihood distribution of the $k$ output classes.

Hence, the output layer uses this function to determine a previous layer output is fits the most relevant data.

$$
p_{i}=\frac{e^{y_{i}}}{\sum_{1}^{k} e^{y_{i}}}
$$

Where $y$ signifies the selected output patch. The selected patch is used in the quantum logicbased weight.

\section{RESULTS AND DISCUSSION}

The proposed Cyber Security Threats detection in IoT utilizing Krill Based Deep Neural Network Stacked Auto Encoders (KDNN-SAE) was executed in the MATLAB platform. Here, the trial results achieved for the introduced method are determined. The exhibition of the introduced Cyber Security Threats detection in IoT is compared to the current Support vector machine (SVM) [26], Naive Bayes (NB) [27], K-nearest neighbourhood (K-NN) [26], and Random Forest (RF) [26] classifiers regarding the accuracy, sensitivity, specificity, precision, recall, f-measure, false-positive rate (FPR), falsenegative rate (FNR) and Kappa statistics. Besides, the introduced work is examined for the 
feature assortment technique and with a feature selection strategy. Analytical processes to analyze the presence of introduced work are specified in the following segment.

\subsection{PERFORMANCE EXAMINATION}

The performance measures of sensitivity, specificity, and accuracy are illustrated in regards to TP, FP, FN, and TN esteem. The proposed technique is examined by the analytical approaches such as accuracy, sensitivity, specificity is stated in conditions (17), (18), (19),

$$
\begin{gathered}
\mathrm{A}^{\prime \prime}=\frac{\left(\overline{\mathrm{t}^{\prime}}{ }_{\mathrm{n}}+\overline{\mathrm{t}}_{\mathrm{p}}{ }_{\mathrm{p}}\right)}{\left(\overline{\mathrm{t}}_{\mathrm{n}}^{\prime}+\overline{\mathrm{t}}_{\mathrm{p}}^{\prime}+\overline{\mathrm{f}}_{{ }_{\mathrm{n}}}+\overline{\mathrm{f}}_{\mathrm{p}}{ }_{\mathrm{p}}\right)} \\
\mathrm{S}_{\mathrm{e}}^{\prime \prime}=\overline{\mathrm{t}}_{\mathrm{p}}^{\prime} /\left(\overline{\mathrm{t}}_{\mathrm{p}}^{\prime}+\overline{\mathrm{f}}_{\mathrm{n}}^{\prime}{ }_{\mathrm{n}}\right) \\
\mathrm{S}_{\mathrm{p}}^{\prime \prime}=\overline{\mathrm{t}}_{\mathrm{n}}^{\prime} /\left(\overline{\mathrm{t}}_{\mathrm{n}}{ }_{\mathrm{n}}+\overline{\mathrm{f}}_{\mathrm{n}}{ }\right)
\end{gathered}
$$

Where $A^{\prime \prime}$ denotes an accuracy, $S_{e}^{\prime \prime}$ denotes sensitivity, $S_{p}^{\prime \prime}$ denotes a specificity, $\overline{\mathrm{t}}_{\mathrm{n}}^{\prime}$ signifies a true negative, $\overline{\mathrm{t}}_{\mathrm{p}}^{\prime}$ signifies a true positive, $\overline{\mathrm{f}}_{\mathrm{p}}^{\prime}$ signifies a false positive, and $\overline{\mathrm{f}}_{\mathrm{n}}$ signifies a false negative.

The comparison analysis of the proposed KDNN-SAE with existing SVM, NB, K$\mathrm{NN}$, and RF classifiers concerning the accuracy, sensitivity, and specificity is given. Here, figure 5 represents the comparison analysis without feature selection and figure 6 represents the comparison analysis with the feature selection process.

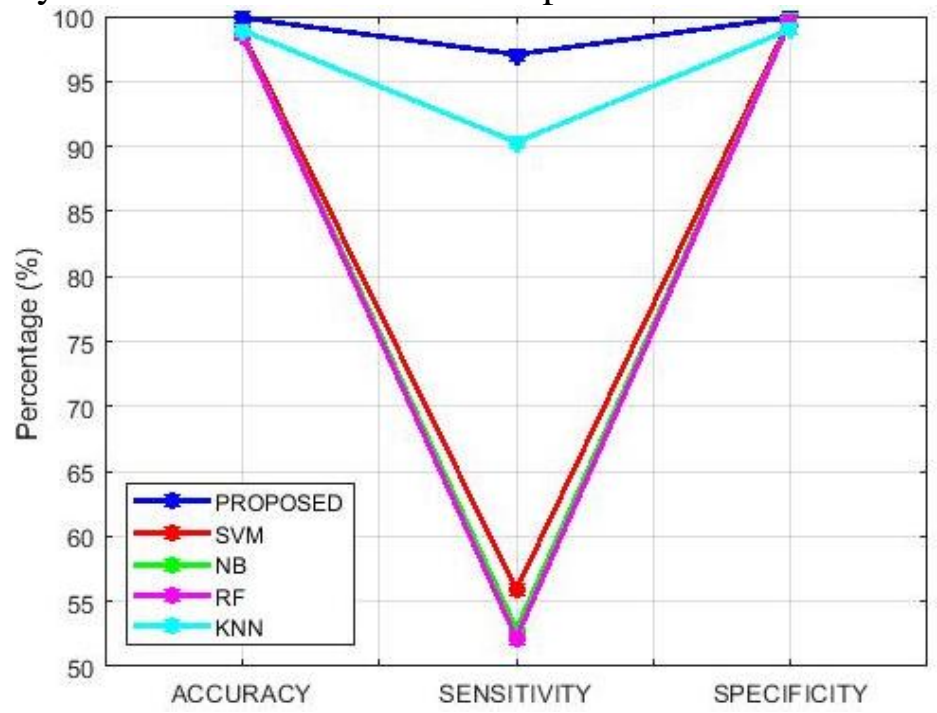

Figure 5: Comparison analysis of accuracy, sensitivity, specificity without feature selection 


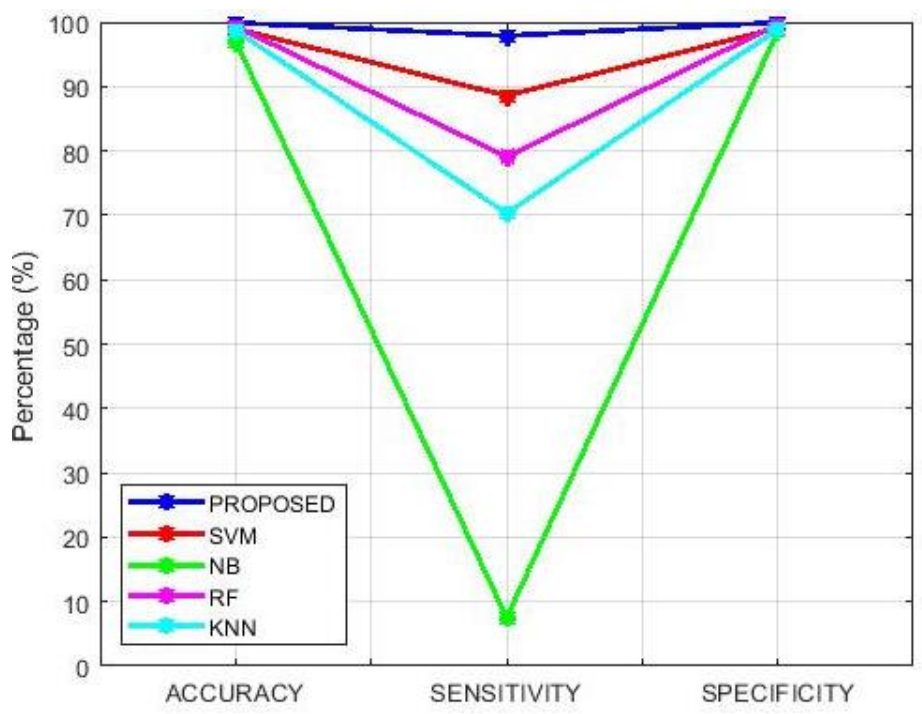

Figure 6: Comparison analysis of accuracy, sensitivity, specificity with feature selection Figure $5 \&$ figure 6 illustrates the proposed KDNN-SAE gives better performance than the existing SVM, K-NN, NB, and RF classifications. The comparison analysis of the proposed with earlier techniques in terms of many statistical measures with feature selection is given in table 1.

Table 1: Comparison analysis of proposed technique with features selection

\begin{tabular}{cccccccccc}
\hline \hline Techniques & Accuracy & Sensitivity & Specificity & precision & Recall & $\begin{array}{c}\text { f- } \\
\text { measure }\end{array}$ & FPR & FNR & $\begin{array}{c}\text { Kappa } \\
\text { statistics }\end{array}$ \\
\hline \hline Proposed & 0.99 & 0.97 & 0.99 & 0.95 & 0.97 & 0.96 & 0.0006 & 0.02 & 0.99 \\
SVM & 0.98 & 0.88 & 0.99 & 0.38 & 0.88 & 0.53 & 0.009 & 0.11 & 0.98 \\
NB & 0.97 & 0.07 & 0.98 & 0.08 & 0.07 & 0.07 & 0.014 & 0.92 & 0.96 \\
RF & 0.99 & 0.79 & 0.99 & 0.76 & 0.79 & 0.77 & 0.003 & 0.20 & 0.99 \\
KNN & 0.98 & 0.70 & 0.98 & 0.29 & 0.70 & 0.41 & 0.01 & 0.29 & 0.98 \\
\hline \hline
\end{tabular}

\subsubsection{F-measure}

The harmonic mean of precision and recall is F-measure. This measures the test result's accuracy. The F-measure takes its best value at 1 followed by worst at 0 . It is calculated by the equation (20).

$$
F_{\text {measure }}=2 *(\text { precision } * \text { recall }) /(\text { precision }+ \text { recall })
$$

\subsubsection{Precision}

Precision is the related projected data divided by projected data by the classifier.

\subsubsection{Recall}

$$
\text { Precision }=\overline{\mathrm{t}}_{\mathrm{p}}^{\prime} / \overline{\mathrm{t}}_{\mathrm{p}}^{\prime}+\overline{\mathrm{f}}_{\mathrm{p}}
$$

The recall is the related projected data divided by the sum of related samples that corresponds to a certain group present in the database.

$$
\text { Re call }=\overline{\mathrm{t}}_{\mathrm{p}}^{\mathrm{p}_{\mathrm{t}}} / \overline{\mathrm{t}}_{\mathrm{p}}+\overline{\mathrm{f}}_{\mathrm{p}}
$$

The comparison analysis of proposed KDNN-SAE with existing SVM, NB, K-NN, and RF classifiers without $\&$ with feature selection is given in figure 7 and figure 8 . 


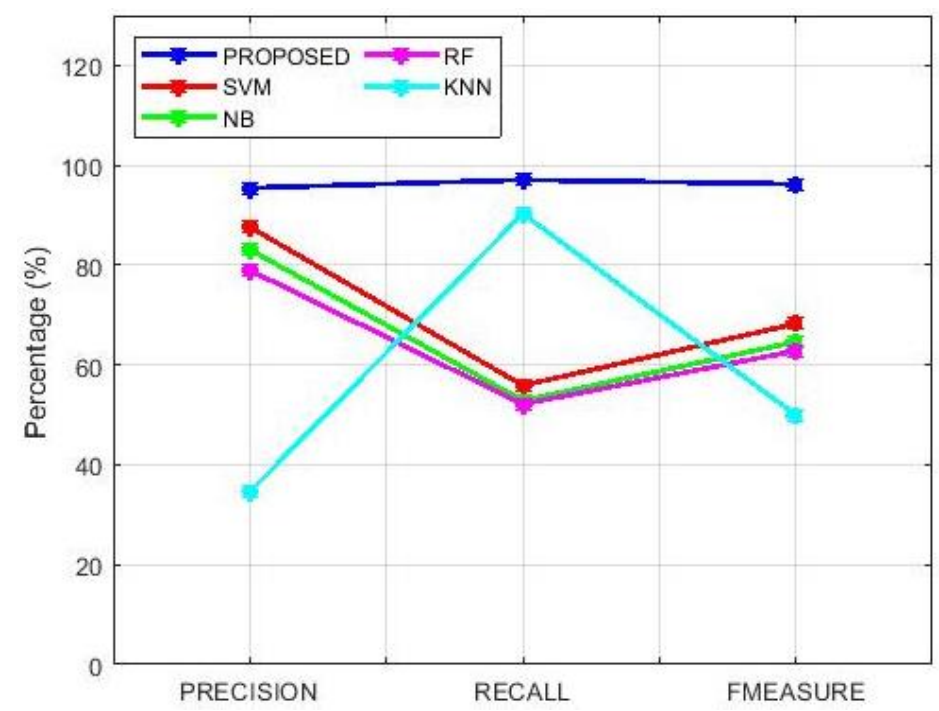

Figure 7: Comparison analysis of precision, recall, F-measure without feature selection

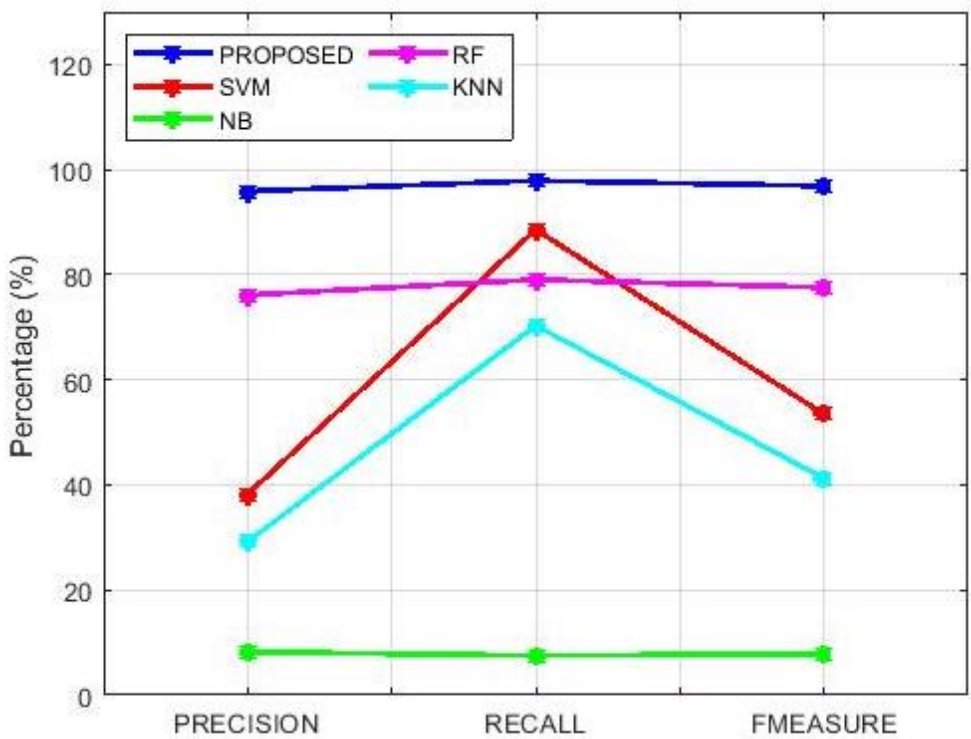

Figure 8: Comparison analysis of precision, recall, F-measure with feature selection

Figure $7 \&$ figure 8 illustrates the proposed KDNN-SAE provides better performance than the existing SVM, K-NN, NB, and RF classifications. The comparison analysis of the proposed with earlier techniques in expressions of various performance measures without feature selection is given in table 2.

Table 2: Comparison analysis of proposed technique without features selection

\begin{tabular}{cccccccccc}
\hline \hline Methods & Accuracy & Sensitivity & Specificity & precision & Recall & $\begin{array}{c}\text { f- } \\
\text { measure }\end{array}$ & FPR & FNR & $\begin{array}{c}\text { Kappa } \\
\text { statistics }\end{array}$ \\
\hline \hline Proposed & 0.99 & 0.97 & 0.99 & 0.95 & 0.97 & 0.96 & 0.0006 & 0.029 & 0.99 \\
SVM & 0.98 & 0.55 & 0.99 & 0.87 & 0.55 & 0.68 & 0.001 & 0.44 & 0.98 \\
NB & 0.98 & 0.52 & 0.99 & 0.83 & 0.52 & 0.64 & 0.002 & 0.47 & 0.98 \\
RF & 0.98 & 0.52 & 0.99 & 0.79 & 0.52 & 0.62 & 0.003 & 0.47 & 0.98 \\
KNN & 0.98 & 0.90 & 0.99 & 0.34 & 0.90 & 0.5 & 0.009 & 0.09 & 0.98 \\
\hline \hline
\end{tabular}

\subsubsection{FPR}

FPR is established as the fraction of some negatives wrongly dignified as positives out of the sum of real negatives. FPR is calculated employing the equation (23). 


$$
F P R=\frac{\overline{\mathrm{f}}_{\mathrm{p}}}{{\overline{\mathrm{f}^{\prime}}}_{\mathrm{p}}+\overline{\mathrm{t}}_{\mathrm{n}}^{\prime}}
$$

The comparison analysis of proposed KDNN-SAE in conditions of false-positive rate without and with feature selection is shown in figure 9 and figure 10.

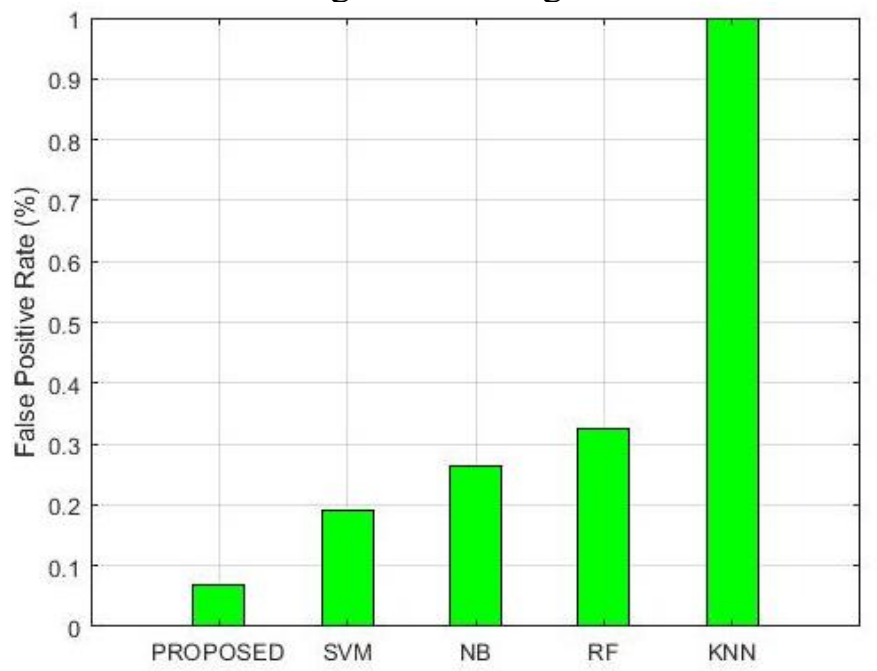

Figure 9: Comparison analysis of false-positive rate without feature selection

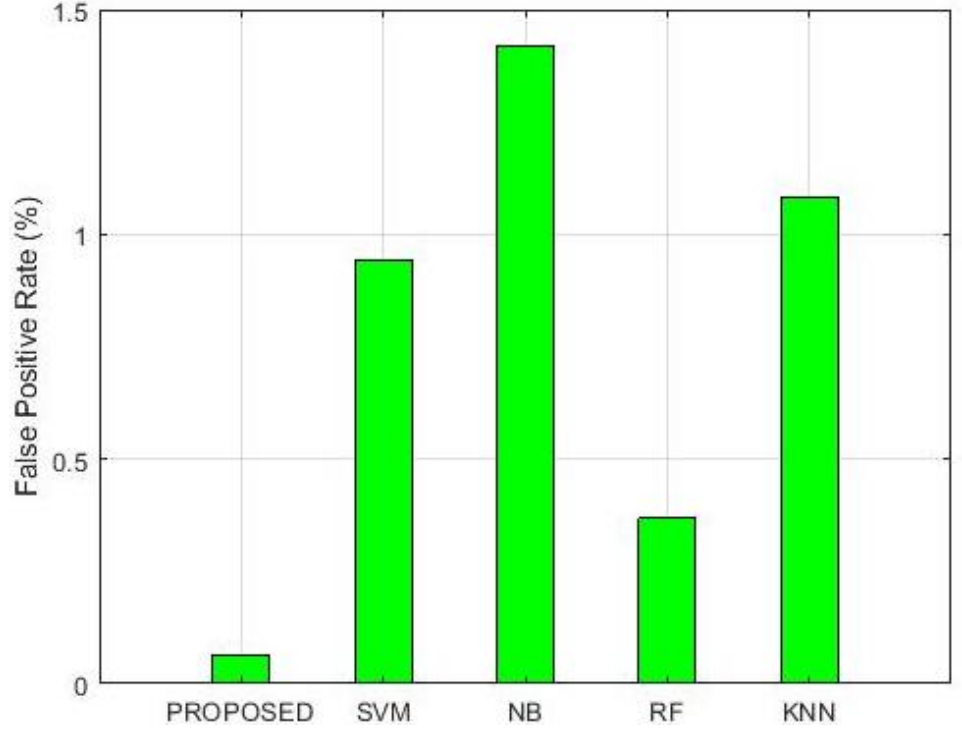

Figure 10: Comparison analysis of false-positive rate with feature selection

Figure $9 \&$ figure 10 illustrates the proposed KDNN-SAE gives better performance than the existing SVM, K-NN, NB, and RF classifications.

\subsubsection{FNR}

FPR is predictable as the fraction of some positives wrongly distinguished as negatives. It is calculated employing the equation (24).

$$
F N R=\frac{\overline{\mathrm{f}}_{\mathrm{n}}}{\overline{\mathrm{f}}_{\mathrm{n}}+\overline{\mathrm{t}}_{\mathrm{p}}^{\prime}}
$$

The comparison analysis of the proposed KDNN-SAE in terms of false negative rate without and with feature selection is given in figure 11 and figure 12. 


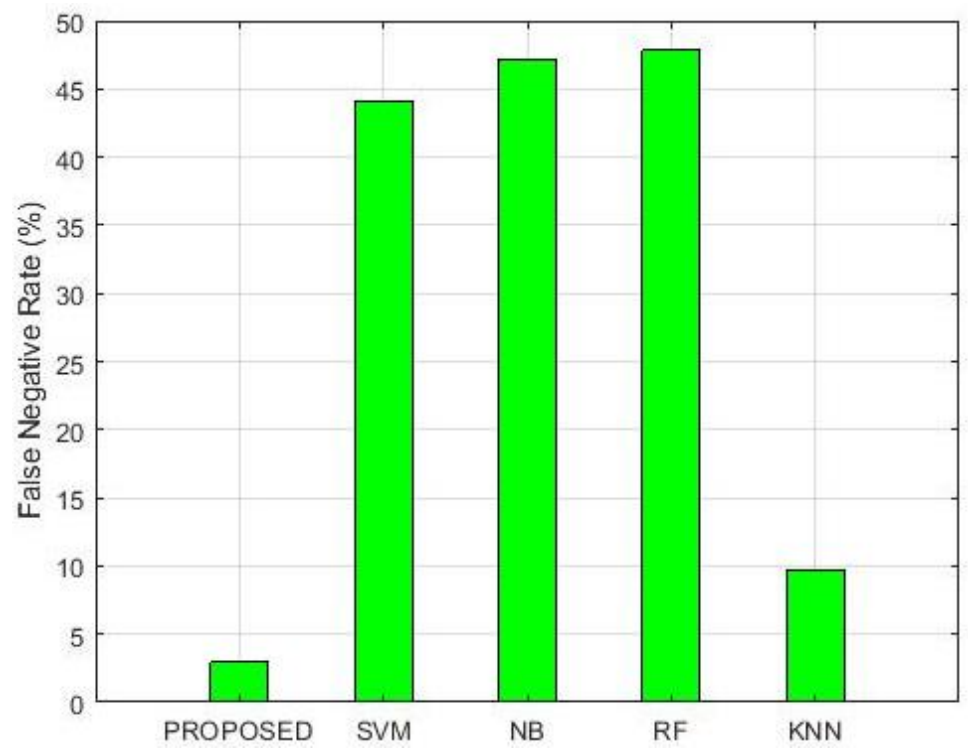

Figure 11: Comparison analysis of false-negative rate without feature selection

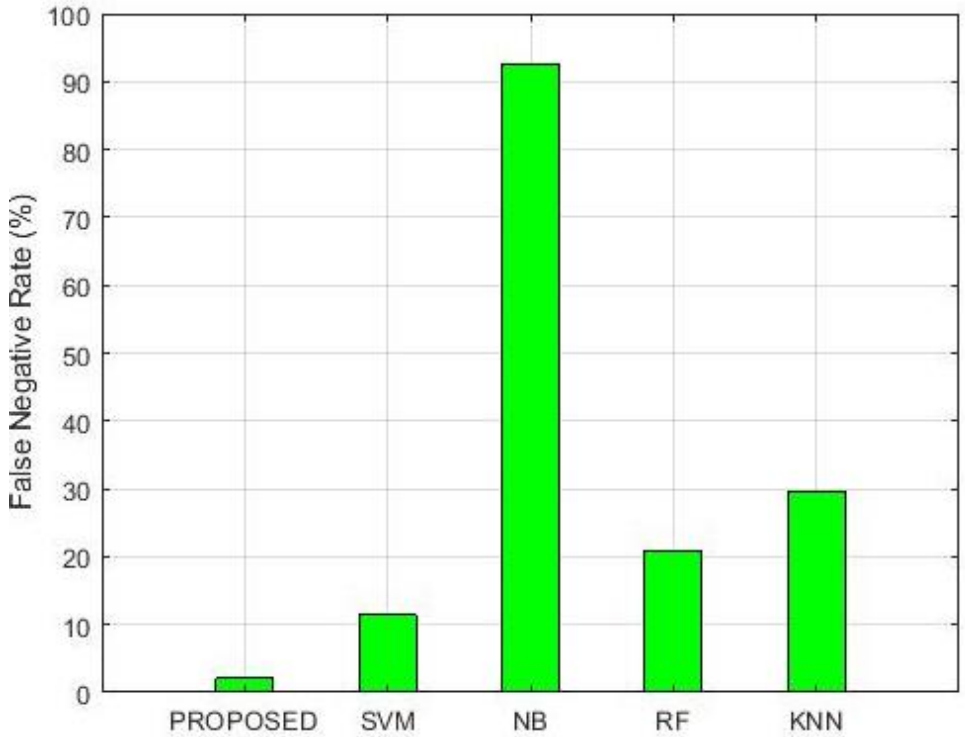

Figure 12: Comparison analysis of false-negative rate with feature selection Figure $11 \&$ figure 12 illustrates the proposed KDNN-SAE gives better performance than the existing SVM, K-NN, NB, and RF classifications.

\subsubsection{Kappa Statistics value}

Measures the chance of an agreement between the calculated and the real classes given by,

$$
k=\frac{a_{o}-a_{e}}{1-a_{e}}
$$

Where $a_{o}$ denotes the perceived agreement and $a_{e}$ denotes the predictable agreement. The comparison analysis of proposed KDNN-SAE in conditions of kappa statistics without and with feature selection is shown in figure 13 and figure 14 , 


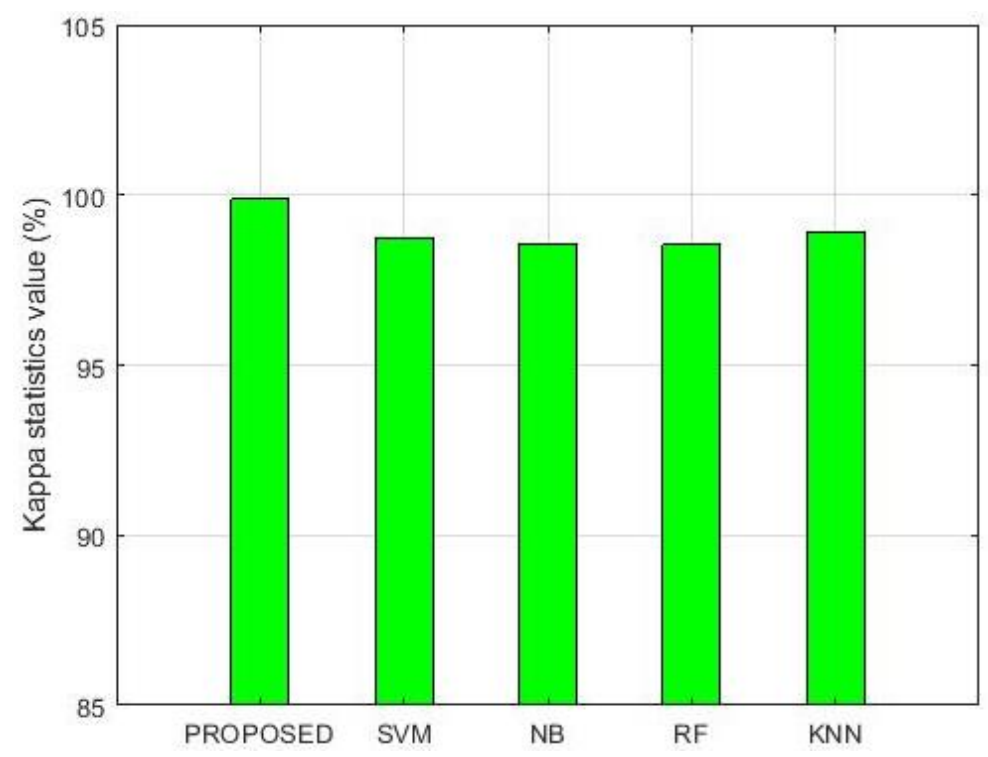

Figure 13: Comparison graph in terms of kappa statistics without feature selection

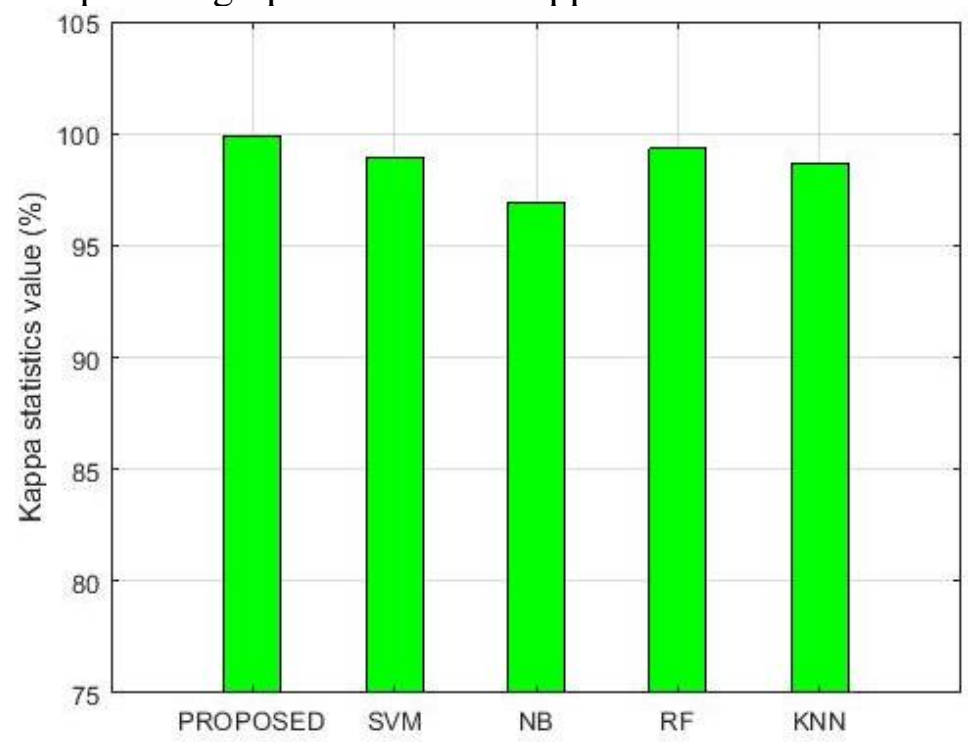

Figure 14: Comparison graph in conditions of kappa statistics with feature selection Figure 13 \& figure 14 illustrates the proposed KDNN-SAE gives better performance than the existing SVM, K-NN, NB, and RF classifications.

\section{CONCLUSION}

This paper introduced Cyber Security Threats detection in IoT utilizing the KDNN-SAE classifier. At first, the dataset is isolated training and testing data independently, and therefore, flow-based features are extricated from the training and testing data. At that point, the extricated features are chosen to utilize the Genetic Algorithm (GA). At last, our methodology completes the execution of the machine learning algorithm KDNN-SAE. The exploratory outcomes demonstrate that our proposed framework performs viably in spoken term recognition. The introduced framework outflanks the current SVM, Naive Bayes, KNN, and RF classifiers regarding the accuracy, sensitivity, specificity, precision, recall, Fmeasure, FPR, FNR, and Kappa statistics.

\section{Compliance with ethical standards}

Compliance with ethical standards Conflict of interest the authors declare that they have no conflict of interest 


\section{REFERENCE}

1. Li, He, Kaoru Ota, and Mianxiong Dong. "Learning IoT in edge: Deep learning for the Internet of Things with edge computing." IEEE Network 32, no. 1 (2018): 96-101.

2. Fadlullah, Zubair Md, Fengxiao Tang, Bomin Mao, Nei Kato, Osamu Akashi, Takeru Inoue, and Kimihiro Mizutani. "State-of-the-art deep learning: Evolving machine intelligence toward tomorrow's intelligent network traffic control systems." IEEE Communications Surveys \& Tutorials 19, no. 4 (2017): 2432-2455

3. Kato, Nei, Zubair Md Fadlullah, Bomin Mao, Fengxiao Tang, Osamu Akashi, Takeru Inoue, and Kimihiro Mizutani. "The deep learning vision for heterogeneous network traffic control: Proposal, challenges, and future perspective." IEEE wireless communications 24, no. 3 (2016): 146-153.

4. Diro, Abebe Abeshu, and Naveen Chilamkurti. "Distributed attack detection scheme using deep learning approach for the Internet of Things." Future Generation Computer Systems 82 (2018): 761-768.

5. Grammatikis, Panagiotis I. Radoglou, Panagiotis G. Sarigiannidis, and Ioannis D. Moscholios. "Securing the Internet of Things: Challenges, threats, and solutions." Internet of Things 5 (2019): 41-70.

6. Zhou, Yiyun, Meng Han, Liyuan Liu, Jing Selena He, and Yan Wang. "Deep learning approach for cyberattack detection." In IEEE INFOCOM 2018-IEEE Conference on Computer Communications Workshops (INFOCOM WKSHPS), pp. 262-267. IEEE, 2018.

7. Bertino, Elisa, and Nayeem Islam. "Botnets and internet of things security." Computer 50, no. 2 (2017): 76-79.

8. Kumar, S., L. Madhavan, M. Nagappan, and B. Sikdar. "Malware in Pirated Software: Case Study of Malware Encounters in Personal Computers." In 2016 11th International Conference on Availability, Reliability, and Security (ARES), pp. 423427. IEEE, 2016.

9. Eder-Neuhauser, Peter, Tanja Zseby, Joachim Fabini, and Gernot Vormayr. "Cyber attack models for smart grid environments." Sustainable Energy, Grids and Networks 12 (2017): 10-29.

10. Tobiyama, Shun, Yukiko Yamaguchi, Hajime Shimada, Tomonori Ikuse, and Takeshi Yagi. "Malware detection with deep neural network using process behavior." In 2016 IEEE 40th Annual Computer Software and Applications Conference (COMPSAC), vol. 2, pp. 577-582. IEEE, 2016.

11. Han, Shanshan, Fu Ren, Chao Wu, Ying Chen, Qingyun Du, and Xinyue Ye. "Using the tensorflow deep neural network to classify mainland china visitor behaviours in hong kong from check-in data." ISPRS International Journal of Geo-Information 7, no. 4 (2018): 158.

12. Yang, Yanqing, Kangfeng Zheng, Chunhua Wu, and Yixian Yang. "Improving the classification effectiveness of intrusion detection by using improved conditional variational autoencoder and deep neural network." Sensors 19, no. 11 (2019): 2528.

13. Campos, Víctor, Francesc Sastre, Maurici Yagües, Jordi Torres, and Xavier Giró-iNieto. "Scaling a convolutional neural network for classification of adjective noun pairs with tensorflow on gpu clusters." In 2017 17th IEEE/ACM International Symposium on Cluster, Cloud and Grid Computing (CCGRID), pp. 677-682. IEEE, 2017.

14. Abadi, Martín, Paul Barham, Jianmin Chen, Zhifeng Chen, Andy Davis, Jeffrey Dean, Matthieu Devin et al. "Tensorflow: A system for large-scale machine learning." 
In 12th \{USENIX\} symposium on operating systems design and implementation (\{OSDI\} 16), pp. 265-283. 2016.

15. Mirza, Olfat M., and Mike Joy. "Style analysis for source code plagiarism detection." PhD diss., University of Warwick, Coventry, UK, 2018.

16. Ullah, Farhan, Junfeng Wang, Sohail Jabbar, Fadi Al-Turjman, and Mamoun Alazab. "Source Code Authorship Attribution Using Hybrid Approach of Program Dependence Graph and Deep Learning Model." IEEE Access 7 (2019): 141987141999.

17. Gorshkov, Sergey, Maxim Nered, Eugene Ilyushin, Dmitry Namiot, and Vladimir Sukhomlin. "Source Code Authorship Identification Using Tokenization and Boosting Algorithms." In International Conference on Modern Information Technology and IT Education, pp. 295-308. Springer, Cham, 2018.

18. Alrabaee, Saed, Paria Shirani, Mourad Debbabi, and Lingyu Wang. "On the feasibility of malware authorship attribution." In International Symposium on Foundations and Practice of Security, pp. 256-272. Springer, Cham, 2016.

19. Wang, Wei, Mengxue Zhao, and Jigang Wang. "Effective android malware detection with a hybrid model based on deep autoencoder and convolutional neural network." Journal of Ambient Intelligence and Humanized Computing 10, no. 8 (2019): 3035-3043.

20. Tobiyama, Shun, Yukiko Yamaguchi, Hajime Shimada, Tomonori Ikuse, and Takeshi Yagi. "Malware detection with deep neural network using process behavior." In 2016 IEEE 40th Annual Computer Software and Applications Conference (COMPSAC), vol. 2, pp. 577-582. IEEE, 2016.

21. Lee, Jonghoon, Jonghyun Kim, Ikkyun Kim, and Kijun Han. "Cyber Threat Detection Based on Artificial Neural Networks Using Event Profiles." IEEE Access 7 (2019): $165607-165626$.

22. Yin, Chuanlong, Yuefei Zhu, Jinlong Fei, and Xinzheng He. "A deep learning approach for intrusion detection using recurrent neural networks." Ieee Access 5 (2017): 21954-21961.

23. Cui, Zhihua, Fei Xue, Xingjuan Cai, Yang Cao, Gai-ge Wang, and Jinjun Chen. "Detection of malicious code variants based on deep learning." IEEE Transactions on Industrial Informatics 14, no. 7 (2018): 3187-3196.

24. M. Choraś and R. Kozik, "Machine learning techniques applied to detect cyber attacks on web applications," in Logic Journal of the IGPL, vol. 23, no. 1, pp. 45-56, Feb. 2015, doi: 10.1093/jigpal/jzu038.

25. Meng, Fanzhi, Fang Lou, Yunsheng Fu, and Zhihong Tian. "Deep learning based attribute classification insider threat detection for data security." In 2018 IEEE Third International Conference on Data Science in Cyberspace (DSC), pp. 576-581. IEEE, 2018.

26. Saharkhizan, Mahdis, et al. "An Ensemble of Deep Recurrent Neural Networks for Detecting IoT Cyber Attacks Using Network Traffic." IEEE Internet of Things Journal (2020).

27. Vinayakumar, R., et al. "Deep learning approach for intelligent intrusion detection system." IEEE Access 7 (2019): 41525-41550.

28. Lashkari, A. H., Draper-Gil, G., Mamun, M. S. I., \& Ghorbani, A. A. (2017, February). Characterization of tor traffic using time based features. In ICISSp (pp. 253-262).

29. Hamdani, Tarek M., Adel M. Alimi, and Fakhri Karray. "Distributed genetic algorithm with bi-coded chromosomes and a new evaluation function for features 
selection." 2006 IEEE International Conference on Evolutionary Computation. IEEE, 2006. 
Figures

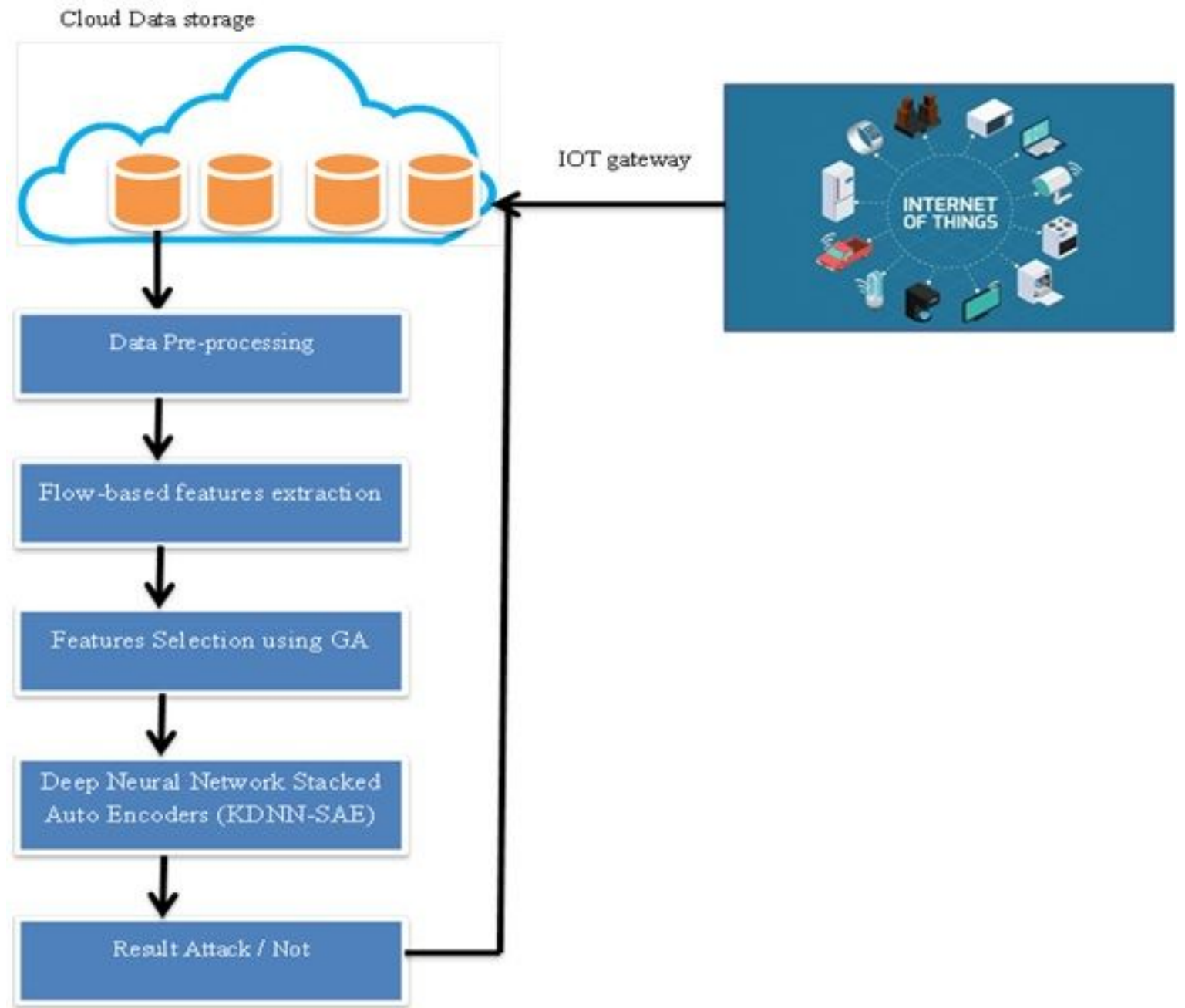

Figure 1

The illustration of the presented approach

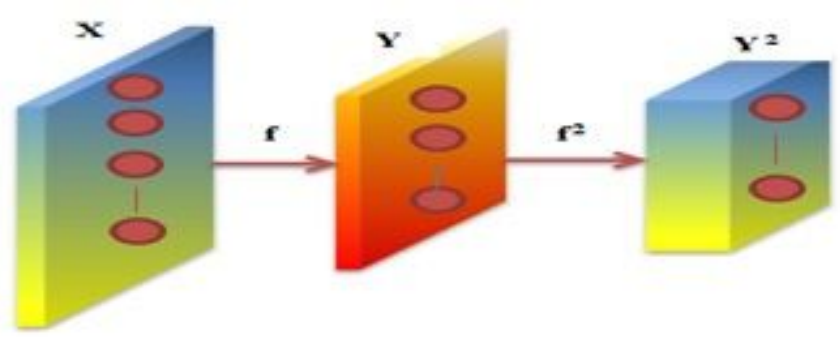

Figure 2

Structure of SDAE 


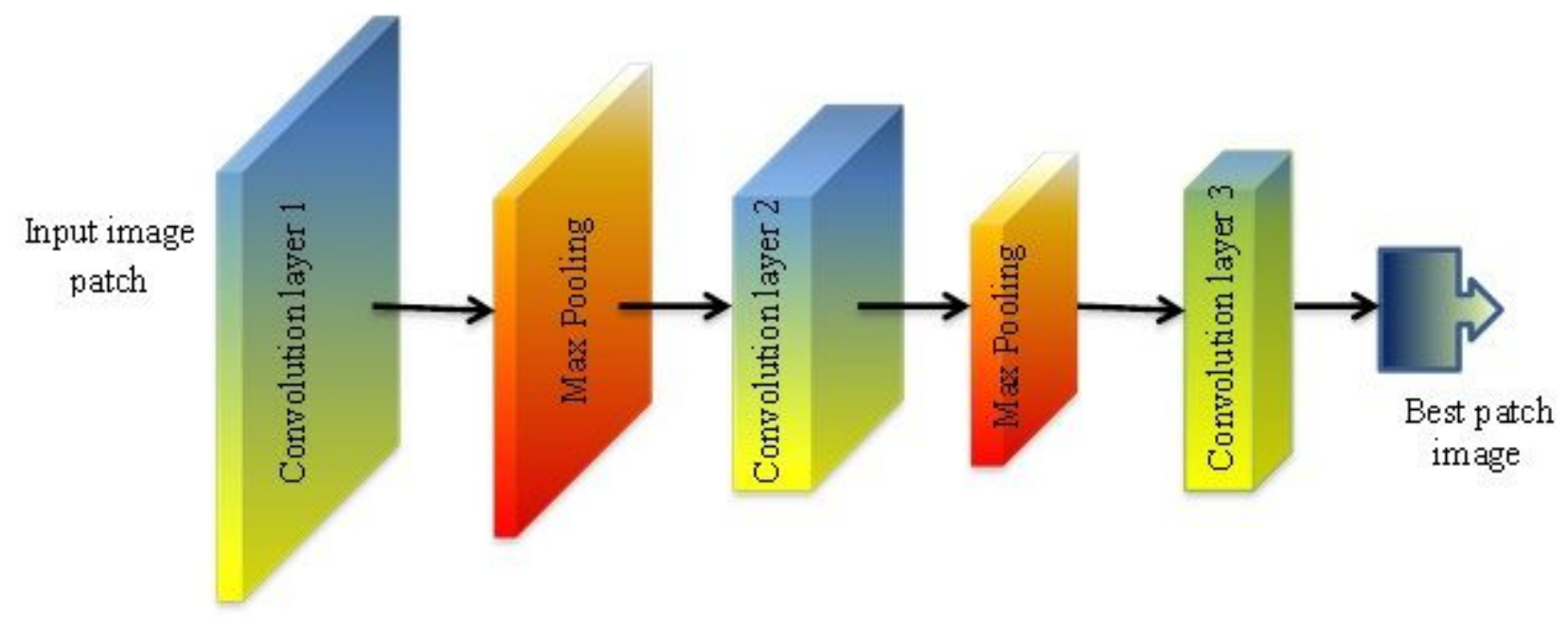

Figure 3

Presented KDNN-SAE system

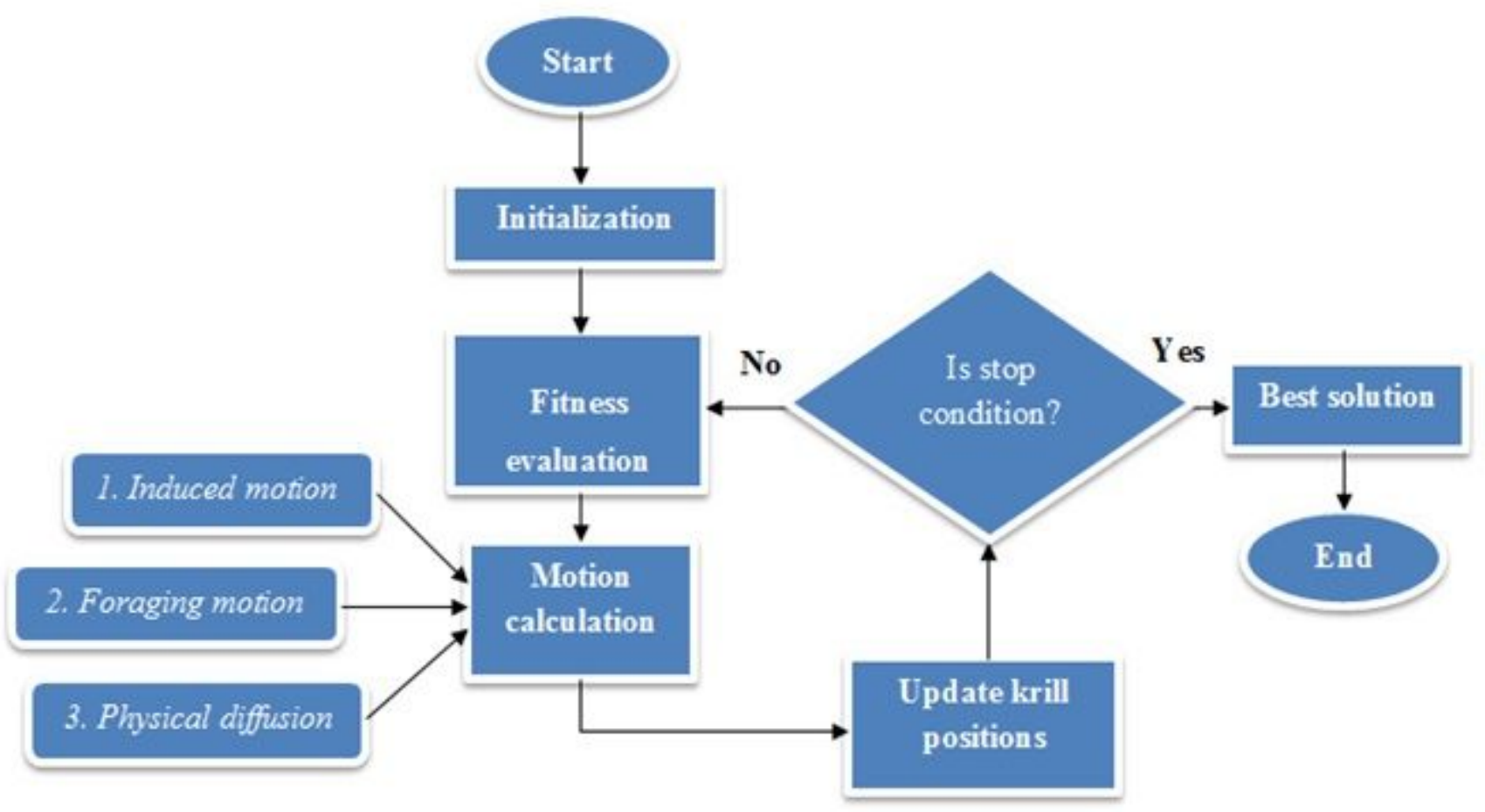

Figure 4

Flow representation of $\mathrm{KHO}$ 


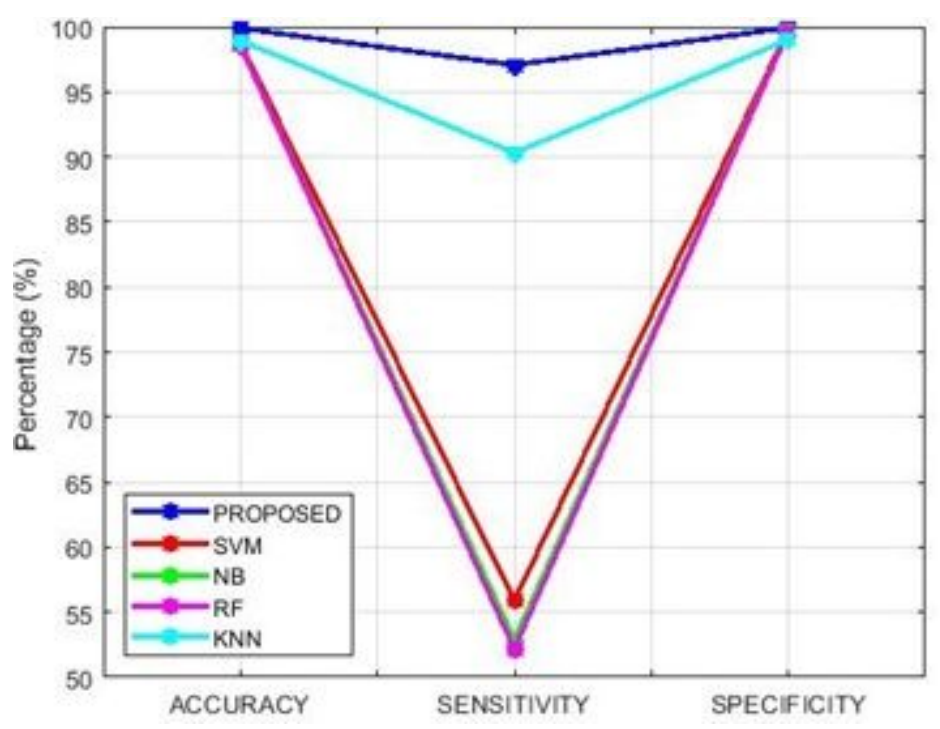

Figure 5

Comparison analysis of accuracy, sensitivity, specificity without feature selection

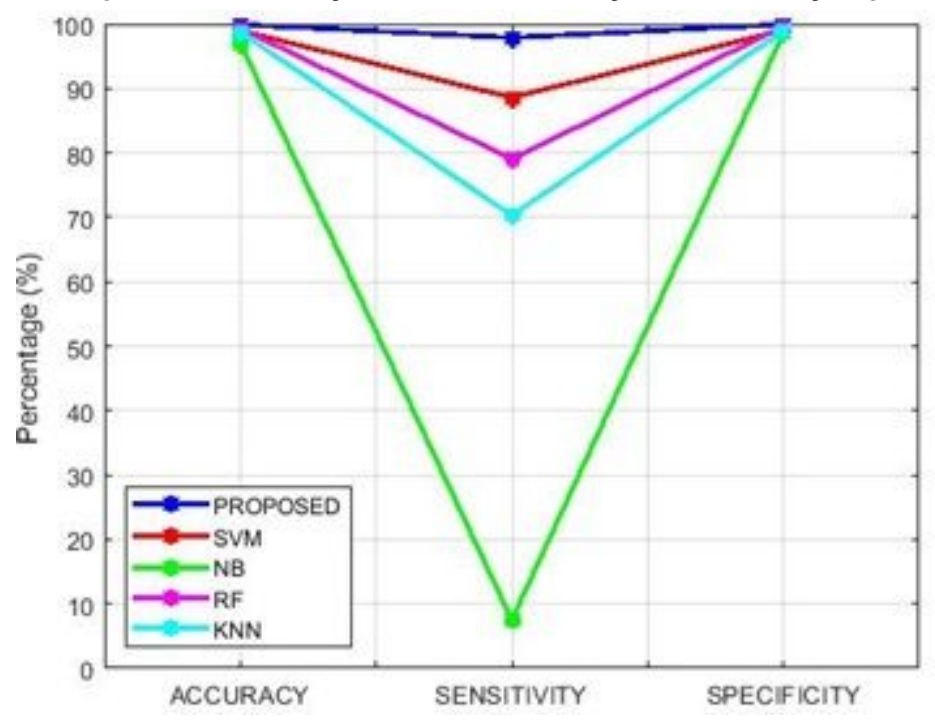

Figure 6

Comparison analysis of accuracy, sensitivity, specificity with feature selection 


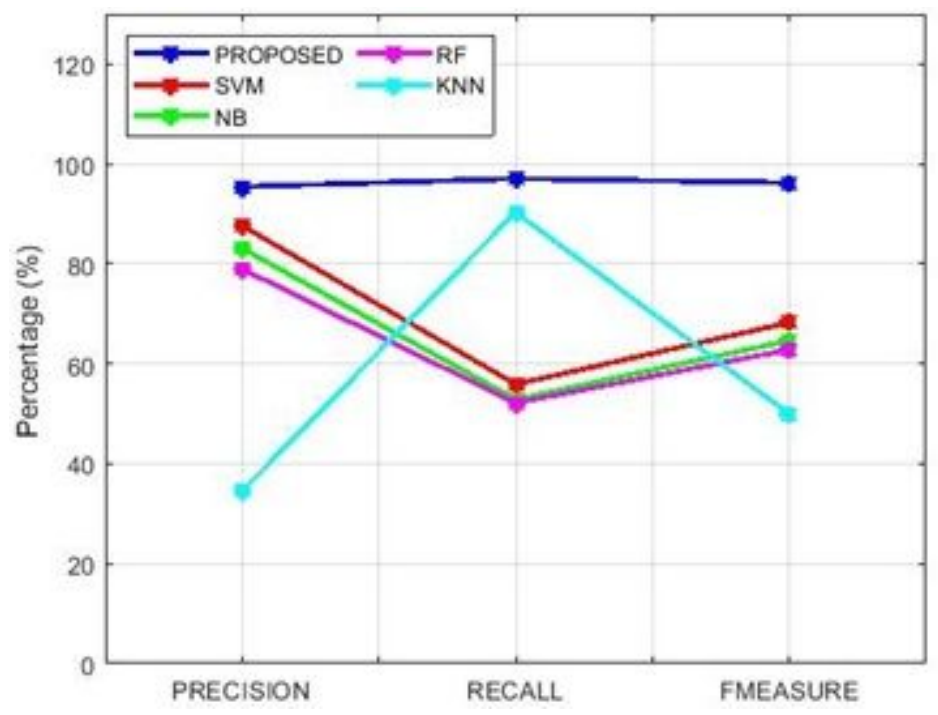

Figure 7

Comparison analysis of precision, recall, F-measure without feature selection

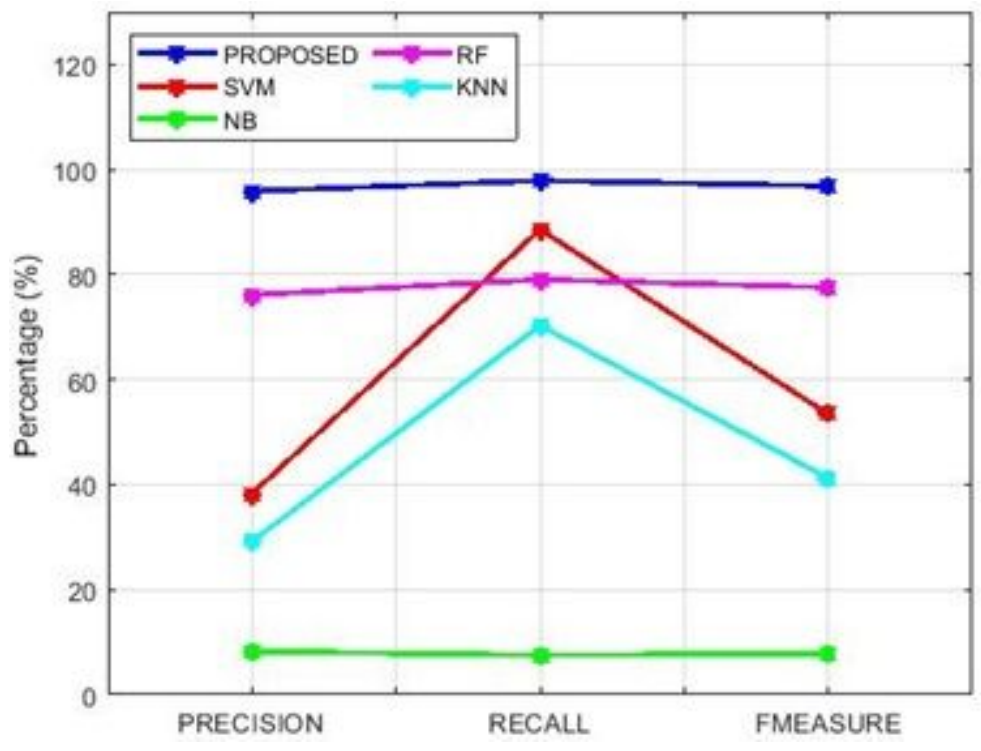

Figure 8

Comparison analysis of precision, recall, F-measure with feature selection 


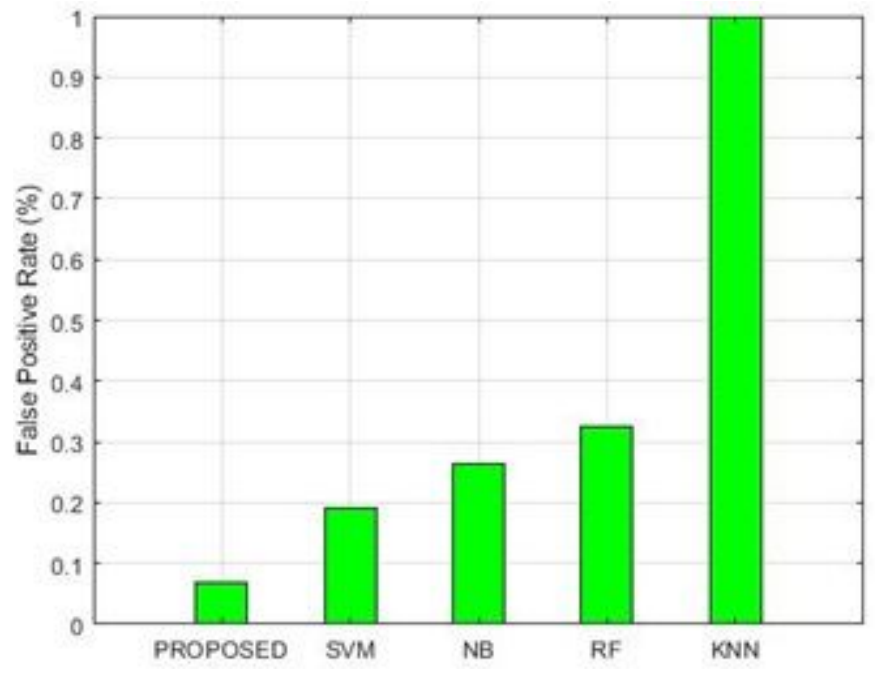

Figure 9

Comparison analysis of false-positive rate without feature selection

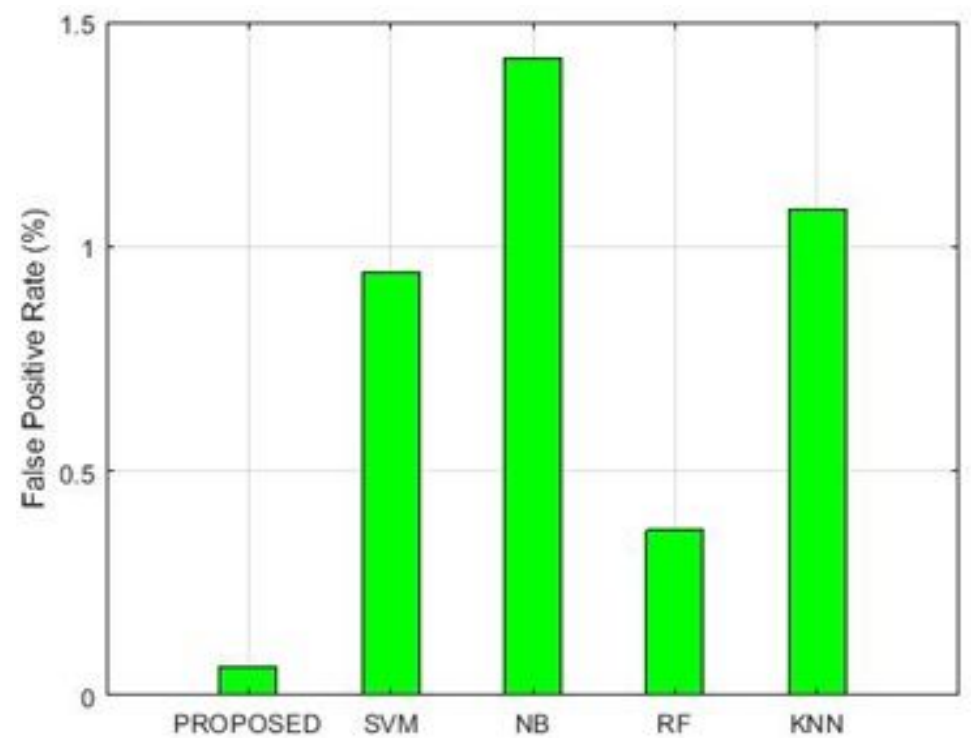

Figure 10

Comparison analysis of false-positive rate with feature selection 


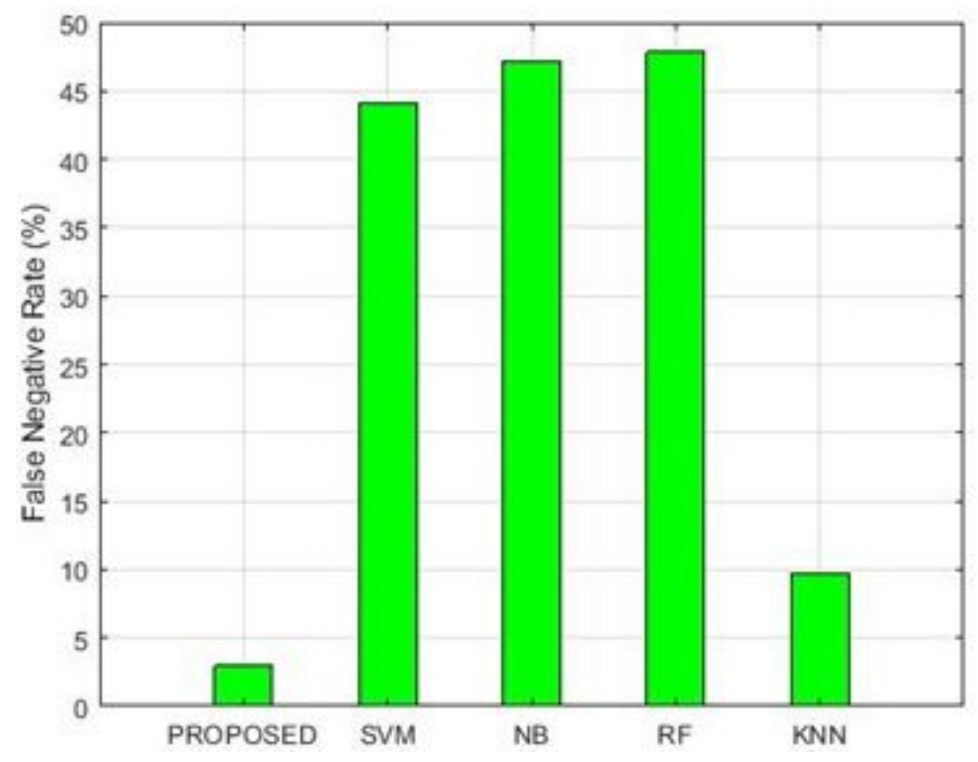

Figure 11

Comparison analysis of false-negative rate without feature selection

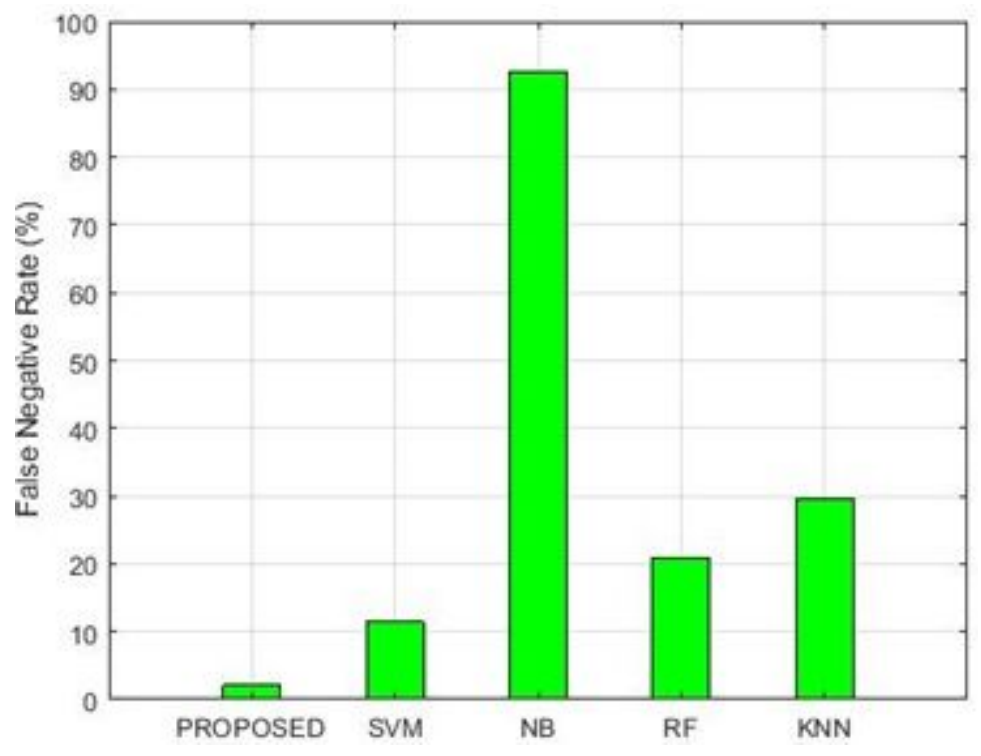

Figure 12

Comparison analysis of false-negative rate with feature selection 


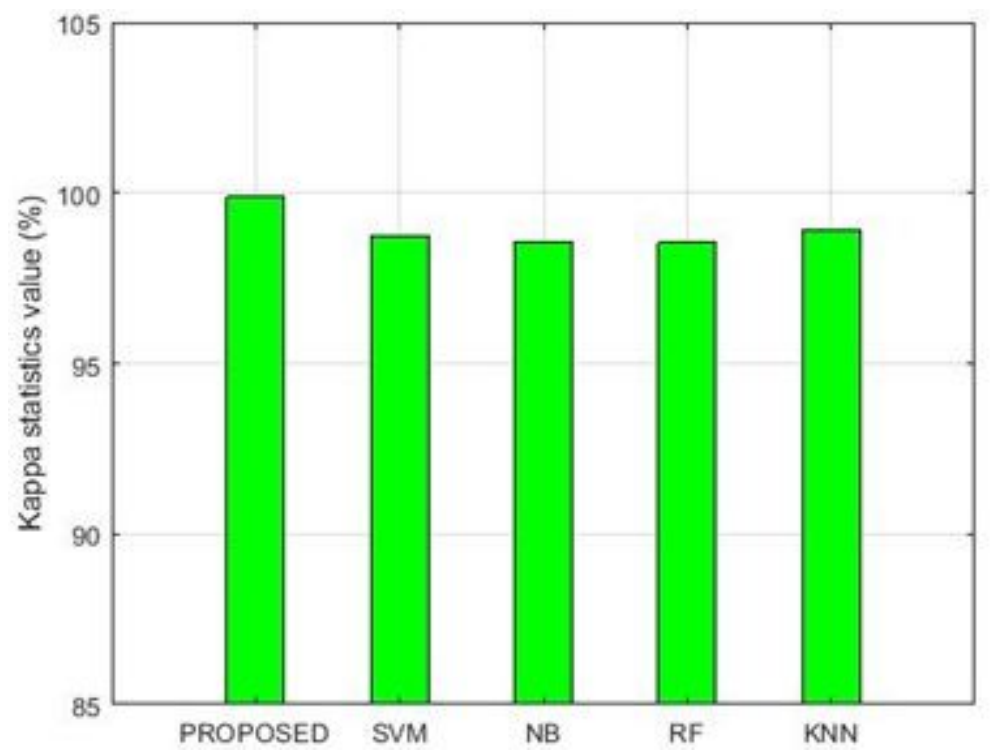

Figure 13

Comparison graph in terms of kappa statistics without feature selection

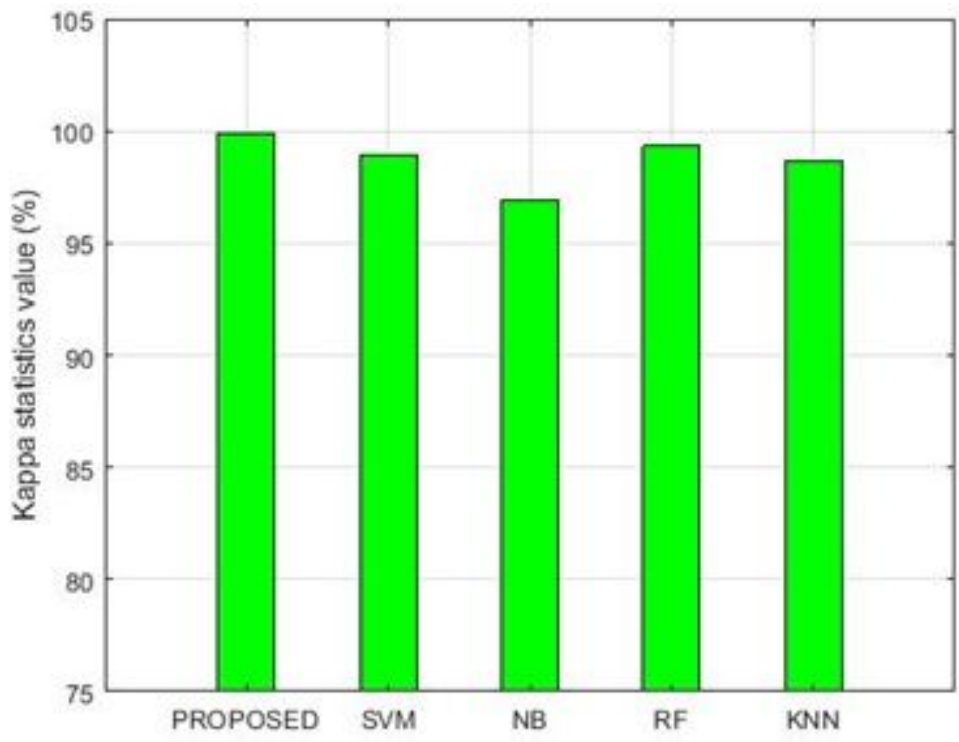

Figure 14

Comparison graph in conditions of kappa statistics with feature selection 